\title{
Energy flow analysis of mid-frequency vibration of coupled plate structures with a hybrid analytical wave and finite element model
}

\author{
Yongbin Ma $\mathrm{a}^{\mathrm{a}, \mathrm{b}}$, Yahui Zhang ${ }^{\mathrm{a}^{*}}, \quad$ David Kennedy ${ }^{\mathrm{c}}$
}

${ }^{a}$ State Key Laboratory of Structural Analysis for Industrial Equipment, Faculty of Vehicle Engineering and Mechanics, Dalian University of Technology, Dalian 116023, PR China;

${ }^{b}$ Institute of Advanced Structure Technology, Beijing Institute of Technology, Beijing 100081, PR China

${ }^{c}$ Cardiff School of Engineering, Cardiff University, Cardiff CF24 3AA, Wales, UK

Corresponding author:

Dr. Y. H. Zhang

State Key Laboratory of Structural Analysis for Industrial Equipment

Department of Engineering Mechanics, Faculty of Vehicle Engineering and Mechanics, Dalian University of Technology, Dalian 116023, PR China

Email: zhangyh@dlut.edu.cn

Tel: +8641184706337

Fax: +86411 84708393 


\section{Abstract}

The medium frequency vibration of a built-up plate structure is studied by an energy flow analysis which extends the concept of statistical energy analysis. The propagative waves of the plates are considered as subsystems that carry and spread energy. Symplectic analytical solutions for mode count, modal density and group velocity of each wave subsystem are obtained based on accurate consideration of the plate geometry and boundary conditions, while the joint vibrational behavior is described by a finite element model. The input mobility and coupling factor associated with each wave subsystem are accurately obtained using a hybrid analytical wave and finite element formulation. Based on the power balance relation of each wave subsystem, the system energy equations are established. Numerical examples for built-up structures comprising rectangular plates demonstrate high accuracy and efficiency. In contrast with statistical energy analysis, the energy of each wave subsystem can be obtained, facilitating the understanding and control of structural vibration and local response. The computational time of the hybrid formulation decreases significantly with increasing length/width ratio of the plates. The wave scattering property of the joint can also be obtained and used to replace the finite element model in repetitive analysis.

Keywords: coupled plate structures; mid-frequency vibration; energy flow; symplectic method; finite element method 


\section{Introduction}

In the design of engineering structures, the analysis of vibration and acoustic behavior is of great importance for the evaluation of energy consumption, noise, comfort, safety and fatigue life. In vibro-acoustics, the audio frequency range is conventionally divided into low, medium and high frequency regions. At low frequencies, the structural response shows obvious peak values, and the influence of geometric shape and boundary conditions on the results can be clearly observed. In this range, traditional deterministic methods such as the finite element method (FEM) [1] and the boundary element method (BEM) [2] are appropriate. At high frequencies, smooth response behavior due to modal overlap can be observed and is significantly affected by structural uncertainties. Statistical energy analysis (SEA) [3] is most frequently used in this range. For mid-frequency vibration analysis, the traditional deterministic methods suffer disadvantages of huge computational load and low accuracy, while the statistical methods suffer the limitation that the structural uncertainty is insufficient.

At present, there are three main approaches to the analysis of medium frequency vibration [4]. The first approach is to develop methods with higher efficiency based on the standard FEM or BEM [5-10], or on wave theory [4, 11-17], i.e. extending the range of low frequency deterministic analysis. The second approach is to develop methods based on SEA with more relaxed assumptions [18-21], i.e. extending the range of high frequency statistical analysis. The third approach is to analyze structures by a hybrid framework that combines deterministic methods and statistical methods [22-26].

As an energy flow method, SEA has a great advantage of computational efficiency compared with the displacement based methods when calculating the energy response of 
structures. SEA is said to be an ad hoc extension of the exact results which may be derived for two coupled single degree of freedom oscillators under broadband excitation [27], and also based on many assumptions of high frequency [28]. At medium frequencies, the geometric shape and boundary conditions significantly affect the dynamics of the structure, violating the basic assumption of SEA and the classical asymptotic formulae of its parameters, e.g. modal density, coupling loss factor and input power. Even at high frequencies, the classical formulae of SEA parameters are not appropriate for all structural forms. Therefore, much effort has been made to find more appropriate SEA parameters. Xie et al [29] investigated the effects of boundary conditions on the mode count and modal density of beams and plates using the wavenumber integration method. Seçgin [30] determined SEA parameters for point connected, directly coupled symmetrically laminated composite plates using a numerical modal based approach. Finnveden [31] calculated the modal density, group velocity and coupling loss factor of a waveguide structure using the waveguide FEM. Based on a dual modal formulation and the power injection method, respectively, Totaro et al [32], Bies and Hamid [33] calculated the coupling loss factor used in SEA. Langley and Heron [34] calculated the transmission coefficients and the associated coupling loss factors for a semi-infinite built-up plate structure using the wave dynamic stiffness method. Using FEM to calculate SEA parameters has advantages in handing complex geometry and boundary constraints [35-38].

In this paper, a new energy flow method is proposed for the analysis of mid-frequency vibration of built-up plate structures based on the power balance framework used in SEA. The established concept of using the parameters of input mobility, damping loss factor and coupling loss factor to describe the input, transmission and dissipation of the energy of each 
subsystem in the classical SEA is adopted in the presented energy flow method. In contrast with classical SEA, in which the whole flexural or in-plane wave field of the plates is regarded as a subsystem, each pair of propagative waves of the plates is considered as a subsystem in this paper. Based on this wave subsystem description, the analytical expressions of the SEA parameters can be obtained. Similar ideas have been used in previous studies. For example, the wave intensity analysis proposed by Langley [18] considers the waves in each direction as subsystems based on which the system power balance equations can be established. Based on [39], Wester and Mace [40] adopted analytical waves as subsystems in the SEA of two directly coupled rectangular plates. However, the analytical wave components can only be obtained for plates with two opposite edges simply supported. To overcome this boundary condition limitation the wave FEM $[13,14]$ or the waveguide FEM [31] may be considered. However, because a finite element model is introduced, the advantages in accuracy, efficiency and parametric analysis of the analytical wave description are lost. In this paper, the boundary condition limitation of the traditional analytical wave description is avoided by using the symplectic method [41-45] to describe the vibration behavior of the plates. And then the parameters of input mobility, damping loss factor and coupling loss factor can be obtained using the symplectic analytical waves which exactly reflect the mid-frequency characteristic of the built-up structure. Based on the symplectic analytical wave modes (i.e. wave propagation parameters and wave shapes) of rectangular plates previously obtained by the authors [43, 44], the mode count, modal density and group velocity of each wave subsystem are obtained in symplectic analytical form. Like the coupling loss factor used in the classical SEA, the power transmission between the wave 
subsystems is described by a coupling factor which is finally transferred into the solution of the scattering coefficients associated with the waves of each plate. A hybrid analytical wave and finite element formulation is established by combining the analytical wave description for the plate components and the finite element description for the joint to obtain the input mobility and the scattering property of each wave subsystem with high accuracy and efficiency. Hence, the parameters of the energy flow analysis can be obtained exactly and reflect the mid-frequency characteristics of the structure. Then by considering the power balance the system equations of the energy analysis of the built-up structure are established. Solving the system equations of energy equilibrium directly gives the energy of each wave subsystem. Therefore, compared with SEA, from which only the energy of the whole flexural or in-plane wave field of plates can be obtained, the energy flow analysis presented in this paper can provide more insight into the vibration transmission, and therefore more useful guidance for the control of vibration of the built-up structure.

The paper is organized as follows. Following this introduction, a wave subsystem based description of the transmission of the structural vibration is presented. Then the parameters for the energy flow analysis are derived and the system equations of power balance of the built-up structure are established. Next, the hybrid analytical wave and finite element solution formulation is derived. Finally, the effectiveness of the methods presented is validated based on three kinds of structural forms including a single rectangular plate, two co-planar plates coupled via a structural joint and three angle coupled plates with a structural joint. 


\section{Wave subsystem based description of the vibration transmission}

In previous work $[43,44]$, the authors have investigated the dispersion relation and the harmonic forced vibration response of single plates and directly connected plate assemblies based on symplectical analytical waves. Here the built-up structure comprises rectangular plates connected via a structural joint, as shown in Fig. 1. The vibrational energy of each plate can be considered as being carried and transferred by waves. Hence, the vibration transmitted in the built-up structure can be regarded as the energy exchange between waves belonging to different plate components. The waves of each plate component can be obtained by the symplectic method, and the joint determines the scattering property between the waves. Because only the propagative wave components can effectively transmit energy, only the propagative waves are considered here. Taking the first flexural propagative wave pair of the left plate shown in Fig. 2 as an example to illustrate the power flow between the two plates, the positively travelling wave is $a^{+}$, and the negatively travelling wave is $a^{-}$. Since there is no energy exchange between the wave components of the same plate [42] and the uncoupled plate end is non-dissipative, all of the energy of $a^{-}$is transmitted into $a^{+}$when $a^{-}$injects on the left end of the left plate. When $a^{+}$injects on the right conservative coupling interface, part of the energy of $a^{+}$transmits into the first flexural positively travelling waves of other plates through the joint and the remainder reflects to $a^{-}$. Meanwhile, $a^{-}$also gets energy from the first flexural negatively travelling waves of other plates through the joint. Therefore, the propagative wave components of each plate can be considered as subsystems that carry and spread energy. The total energy of each plate can be obtained by summing the energy of 
each wave subsystem. In contrast to the classical SEA, which divides the vibration of plate into flexural and in-plane subsystems, the energy flow analysis presented here subdivides these two kinds of vibration fields into propagative wave subsystems.

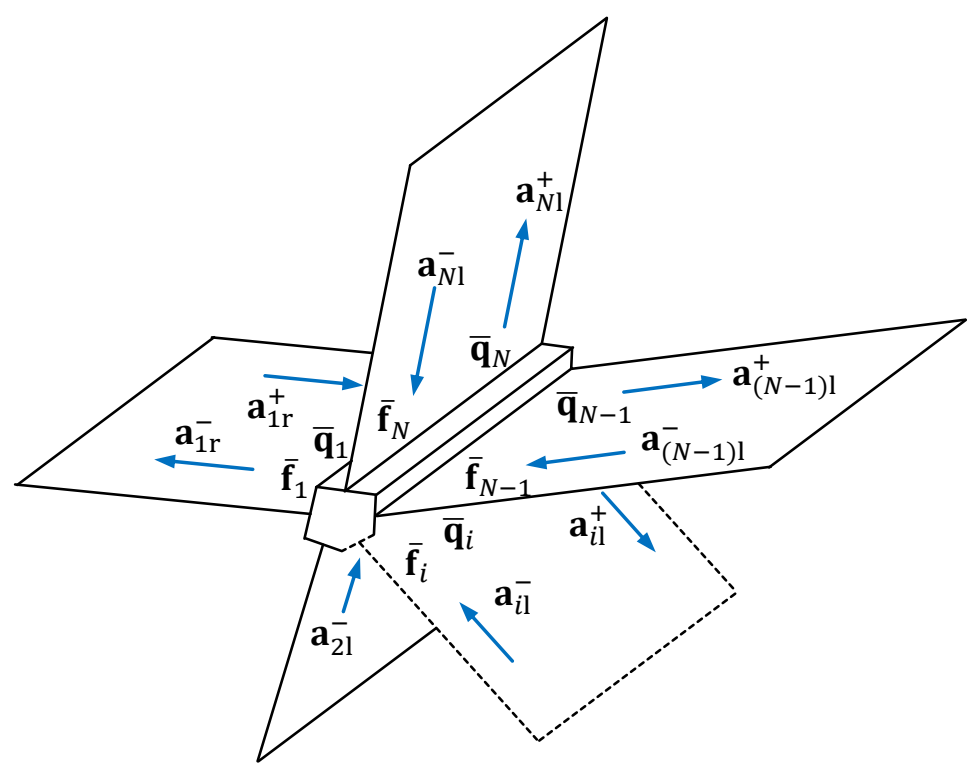

Fig. 1. Schematic of coupled plate structure and wave scattering at the joint, where $\mathbf{a}_{i 1}^{+}, \mathbf{a}_{i 1}^{-}$, $\mathbf{a}_{i \mathrm{r}}^{+}$, and $\mathbf{a}_{i \mathrm{r}}^{-}$are positively and negatively travelling waves at the left and right ends of plate $i$, $\overline{\mathbf{q}}_{i}$ and $\overline{\mathbf{f}}_{\boldsymbol{i}}$ are nodal displacement and internal force vectors at the coupling interface of plate $i(i=1, \ldots, N)$.

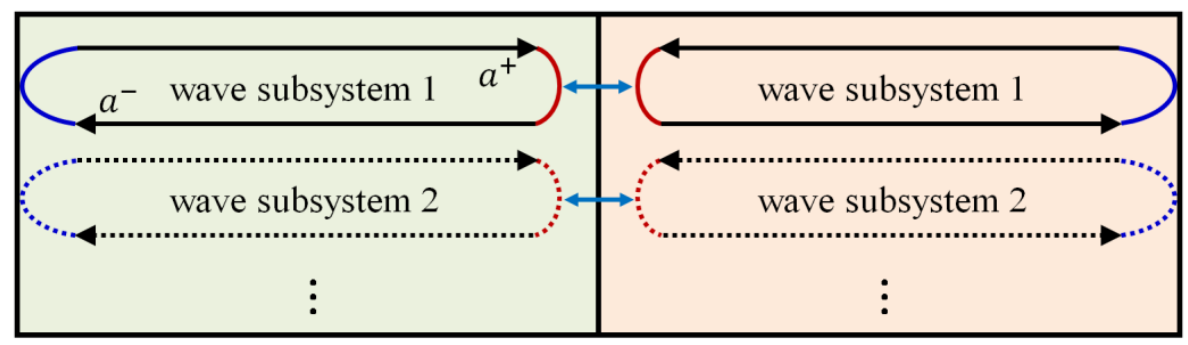

Fig. 2. Schematic of energy transmission between two plates based on propagative wave subsystem description 


\section{Energy flow analysis parameters and system equations of energy balance}

It should be pointed out that, at low and medium frequencies, the in-plane waves are not yet propagative, and so only the energy flow between the flexural propagative waves is considered. For energy flow analysis at high frequencies, the contribution of the in-plane propagative wave subsystems can be easily considered in the system equations of energy balance.

\subsection{Symplectic analytical solution of the mode count}

The propagative wave pairs of rectangular plates are regarded as one-dimensional wave subsystems $i(i=1, \ldots, N)$, for which the mode count can be expressed as [3]

$$
N_{i}\left(k_{y i}\right)=\left(\frac{b}{\pi} k_{y i}+\delta_{\mathrm{BC}}\right)
$$

where $k_{y i}$ is the wave propagation constant for subsystem $i, b$ is the physical length of the subsystem (i.e. the plate length) and $\delta_{\mathrm{BC}}$ is the relevant boundary condition variable. For three types of simple boundary conditions [29]

two ends simply supported

$$
\delta_{\mathrm{BC}}=0
$$

two ends free

$$
\delta_{\mathrm{BC}}=\frac{3}{2}
$$

two ends clamped

$$
\delta_{\mathrm{BC}}=-\frac{1}{2}
$$

Symplectic analytical solutions for the wave propagation parameters $\mu_{y i}$ were given in [43]. The mode count $N_{i}$ of each subsystem is obtained from Eq. (1), using $k_{y i}=i \mu_{y i}(\omega)$ 
and the appropriate value of $\delta_{\mathrm{BC}}$ from Eq. (2). The mode count of the whole flexural vibration field of the plate is then obtained by summing the mode counts of each wave subsystem, i.e.

$$
N=\sum_{i} N_{i}
$$

\subsection{Symplectic analytical solution of the modal density}

Compared with the mode count, the modal density is used more often in the investigation of the dynamic properties of continuous systems. The equation of the flexural wave propagation parameters can be expressed as [43]

$$
f\left(\mu_{y}(\omega), k_{\mathrm{b}}(\omega)\right)=0
$$

where $k_{\mathrm{b}}(\omega)$ is the flexural wavenumber and $\omega$ is the circular frequency. Differentiating Eq. (4) with respect to $\omega$ gives $\frac{\mathrm{d} \mu_{y}}{\mathrm{~d} \omega}=\tilde{f}\left(\mu_{y}(\omega), k_{\mathrm{b}}(\omega)\right)$, based on which the group velocity of the wave subsystem can be obtained as

$$
c_{\mathrm{g}}=\frac{\mathrm{d} \omega}{\mathrm{d} k_{y}}=\frac{\mathrm{d} \omega}{\mathrm{id} \mu_{y}}
$$

Since $\mu_{y}(\omega)$ is a symplectic analytical solution [43], the group velocity obtained here is also symplectic analytical.

The modal density of the $i$ th flexural wave subsystem can be obtained as [3]

$$
n_{i}(\omega)=\frac{\mathrm{d} N_{i}}{\mathrm{~d} \omega}=\frac{b}{\pi} \frac{\mathrm{d} k_{y i}}{\mathrm{~d} \omega}=\frac{b}{\pi} \frac{1}{c_{\mathrm{g} i}}
$$

The total modal density of the whole flexural vibration field is obtained by summing the modal density of each wave subsystem, i.e. 


$$
n(\omega)=\sum_{i} n_{i}(\omega)
$$

The total modal density of the plate can also be calculated by integrating and averaging the mode count obtained in section 3.1 over the associated frequency range, i.e.

$$
n\left(\omega_{j}\right)=\frac{1}{\Delta \omega_{j}} \sum_{i} \int_{\Delta \omega_{j}} N_{i}(\omega) \mathrm{d} \omega
$$

where $\Delta \omega_{j}$ and $\omega_{j}$ are the bandwidth and the central frequency of the $j$ th frequency range, respectively.

As can be seen from Eq. (6), the modal density solution presented here is independent of the boundary conditions on the two ends of the plate. This is a consequence of the transformation of the modal density of a rectangular plate into that of a one-dimensional wave subsystem.

\subsection{Symplectic solution of the coupling factor}

A coupling factor is introduced here to describe the energy exchange between flexural wave subsystems. It is calculated according to the formulation of the coupling loss factor in classical SEA [3] as

$$
\eta_{i j}=\frac{\tau_{i j} c_{\mathrm{g} i} L_{i j}}{\omega \pi A_{i}}
$$

where $L_{i j}$ is the length of the coupling interface, $A_{i}$ is the plate area and $\tau_{i j}$ is the power transmission coefficient associated with each wave. For the parameters $\tau_{i j}$ and $c_{\mathrm{g} i}$ in Eq. (9), solutions which exactly satisfy the plate boundary conditions can be obtained, so the mid-frequency characteristics of the structure can be determined accurately. $\tau_{i j}$ is obtained 
by using the wave scattering coefficient $S_{i j}$, i.e., $\tau_{i j}=\left|S_{i j}\right|^{2}$. The calculation of $S_{i j}$ is described in section 4.4 .

In addition, combining Eq. (6), Eq. (9) and $A_{i}=L_{i j} b_{i}$ gives

$$
n_{i} \eta_{i j}=\frac{b_{i}}{\pi c_{\mathrm{g} i}} \frac{\tau_{i j} c_{\mathrm{g} i} L_{i j}}{\omega \pi A_{i}}=\frac{\tau_{i j}}{\omega \pi^{2}}
$$

and similarly

$$
n_{j} \eta_{j i}=\frac{\tau_{j i}}{\omega \pi^{2}}
$$

The symmetry of the power transmission coefficient $\tau_{i j}$ can be deduced from the symmetry of the wave scattering coefficient $S_{i j}$ [42], so that Eqs. (10) and (11) give the reciprocity relation $n_{i} \eta_{i j}=n_{j} \eta_{j i}$. Therefore, once the coupling factor associated with a wave subsystem is obtained, the corresponding coupling factor of the other wave subsystem can be obtained directly from the reciprocity relationship instead of using Eq. (9).

\subsection{System equations of energy flow balance}

According to the analysis framework of the classical SEA [3], by considering the power equilibrium between the wave subsystems, the system equations of energy balance $E_{i}$ of the whole structure can be expressed as

$$
\left[\begin{array}{cccc}
n_{1} \eta_{1}+n_{1} \sum_{i \neq 1} \eta_{1 i} & -n_{2} \eta_{21} & \cdots & -n_{N} \eta_{N 1} \\
-n_{1} \eta_{12} & n_{2} \eta_{2}+n_{2} \sum_{i \neq 2} \eta_{2 i} & \cdots & -n_{N} \eta_{N 2} \\
\vdots & \vdots & \ddots & \vdots \\
-n_{1} \eta_{1 N} & -n_{2} \eta_{2 N} & \cdots & n_{N} \eta_{N}+n_{N} \sum_{i \neq N} \eta_{N i}
\end{array}\right]\left\{\begin{array}{c}
\frac{E_{1}}{n_{1}} \\
\frac{E_{2}}{n_{2}} \\
\vdots \\
\frac{E_{N}}{n_{N}}
\end{array}\right\}=\frac{1}{\omega}\left\{\begin{array}{c}
\Pi_{1} \\
\Pi_{2} \\
\vdots \\
\Pi_{N}
\end{array}\right\}
$$

or, in matrix form 


$$
\mathbf{C E}=\frac{1}{\omega} \boldsymbol{\Pi}
$$

So far symplectic analytical solutions of the modal density $n_{i}$ and the group velocity $c_{\mathrm{g} i}$ of each wave subsystem have been obtained. The damping loss factors $\eta_{i}$ are generally given. To obtain the coupling factors $\eta_{i j}$ the associated power transmission parameters $\tau_{i j}$ are needed. Therefore, prior to solving Eq. (13), the input power $\Pi_{i}$ from external forces and the wave scattering coefficients $S_{i j}$ associated with each wave subsystem are needed. These two quantities will be obtained by using a hybrid solution formulation in section 4 .

\section{Hybrid analytical wave and finite element solution formulation}

In order to find the input mobility and the power transmission coefficient associated with each wave subsystem, a hybrid analytical wave and finite element analysis is firstly introduced for the built-up structure shown in Fig. 1. The plate components are described by symplectic analytical waves. But, because structural joints in practical engineering applications usually have complex geometry, boundary conditions and material properties associated with specific performance requirements, the joint is described by the FEM.

Also, as shown in Fig.1, the flexural waves associated with each plate component will couple with the in-plane waves at the structural joint. Hence, in order to fully illustrate the effectiveness of the proposed method, the symplectic analytical in-plane waves of the plate must be considered as well as the flexural waves. The authors have previously published the symplectic analysis of the flexural vibration of rectangular plates in detail [43]. Therefore, 
for in-plane vibration, only the symplectic dual equations are given here and the subsequent steps that are exactly the same as for flexural vibration are given in [43].

The symplectic dual equations for the in-plane vibration of rectangular thin plates are

$$
\frac{\partial z}{\partial y}=\mathbf{H z}
$$

where $\mathbf{z}=\left\{\begin{array}{lllll}u & v & T & N\end{array}\right\}^{\mathrm{T}}$ is the state vector; $u, v$ are displacements and $N, T$ are internal forces in the $x$ and $y$ directions, respectively; and the superscript $\mathrm{T}$ indicates the transpose of a vector or a matrix. $\mathbf{H}$ is a Hamiltonian operator matrix with

$$
\mathbf{H}=\left[\begin{array}{cccc}
0 & -\frac{\partial}{\partial x} & -\frac{2(1+v)}{E h} & 0 \\
-v \frac{\partial}{\partial x} & 0 & 0 & -\frac{1-v^{2}}{E h} \\
E h \frac{\partial^{2}}{\partial x^{2}}+\rho h \omega^{2} & 0 & 0 & -v \frac{\partial}{\partial x} \\
0 & \rho h \omega^{2} & -\frac{\partial}{\partial x} & 0
\end{array}\right]
$$

where $E$ is Young's modulus, $h$ is thickness, $\rho$ is density and $v$ is Poisson's ratio.

Next, the displacement-force relations of the plate components and the structural joint at the coupling interfaces are given. Then, by considering the displacement continuity and the equilibrium of the internal forces, the hybrid solution formulation for the built-up structure is established.

\subsection{Displacement-force relation of the joint at the coupling interfaces}

Based on the FEM, the dynamic equilibrium equations of the joint in Fig. 1 can be expressed as

$$
\left[\begin{array}{ll}
\mathbf{D}_{\mathrm{ii}} & \mathbf{D}_{\mathrm{ic}} \\
\mathbf{D}_{\mathrm{ci}} & \mathbf{D}_{\mathrm{cc}}
\end{array}\right]\left\{\begin{array}{l}
\overline{\mathbf{q}}_{\mathrm{i}} \\
\overline{\mathbf{q}}_{\mathrm{c}}
\end{array}\right\}=\left\{\begin{array}{c}
\overline{\mathbf{f}}_{\mathrm{i}} \\
-\overline{\mathbf{f}}_{\mathrm{c}}
\end{array}\right\}
$$


where $\overline{\mathbf{q}}_{\mathrm{c}}=\left[\overline{\mathbf{q}}_{1}^{\mathrm{T}}, \overline{\mathbf{q}}_{2}^{\mathrm{T}}, \ldots, \overline{\mathbf{q}}_{N}^{\mathrm{T}}\right]^{\mathrm{T}}$ is the nodal displacement vector of the coupling interfaces of the joint; $\overline{\mathbf{f}}_{\mathrm{c}}=\left[\overline{\mathbf{f}}_{1}^{\mathrm{T}}, \overline{\mathbf{f}}_{2}^{\mathrm{T}}, \ldots, \overline{\mathbf{f}}_{N}^{\mathrm{T}}\right]^{\mathrm{T}}$ is the nodal force vector applied to the plates by the joint at the coupling interfaces; $\overline{\mathbf{q}}_{\mathrm{i}}$ and $\overline{\mathbf{f}}_{\mathrm{i}}$ are the displacement and external force vectors of the internal degrees of freedom of the joint; and $\mathbf{D}=\left[\begin{array}{ll}\mathbf{D}_{\mathrm{ii}} & \mathbf{D}_{\mathrm{ic}} \\ \mathbf{D}_{\mathrm{ci}} & \mathbf{D}_{\mathrm{cc}}\end{array}\right]$ is the dynamic stiffness matrix of the joint.

Condensing the internal degrees of freedom of the joint onto the interface degrees of freedom, Eq. (16) becomes

$$
\boldsymbol{\alpha} \overline{\mathbf{q}}_{\mathrm{c}}+\boldsymbol{\beta}=\overline{\mathbf{f}}_{\mathrm{c}}
$$

where $\boldsymbol{\alpha}=\mathbf{D}_{\mathrm{ci}} \mathbf{D}_{\mathrm{ii}}^{-1} \mathbf{D}_{\mathrm{ic}}-\mathbf{D}_{\mathrm{cc}}$ and $\boldsymbol{\beta}=-\mathbf{D}_{\mathrm{ci}} \mathbf{D}_{\mathrm{ii}}^{-1} \overline{\mathbf{f}}_{\mathrm{i}}$.

It is noted that $\mathbf{D}_{\mathrm{ii}}^{-1}$ must be evaluated at each frequency, and when the finite element model of the joint is large this inverse operation will significantly increase the computational load. An approximate solution for the inverse operation $\mathbf{D}_{\mathrm{ii}}^{-1}$ can be developed. Based on a modal expansion technique, Eq. (16) can also be expressed as

$$
\left[\begin{array}{ll}
\mathbf{D}_{\mathrm{ii}} & \mathbf{D}_{\mathrm{ic}} \\
\mathbf{D}_{\mathrm{ci}} & \mathbf{D}_{\mathrm{cc}}
\end{array}\right]\left\{\begin{array}{c}
\boldsymbol{\Psi}_{\mathrm{i}} \mathbf{y} \\
\overline{\mathbf{q}}_{\mathrm{c}}
\end{array}\right\}=\left\{\begin{array}{c}
\overline{\mathbf{f}}_{\mathrm{i}} \\
-\overline{\mathbf{f}}_{\mathrm{c}}
\end{array}\right\}
$$

where $\boldsymbol{\Psi}_{\mathrm{i}}$ is the portion of the modal shape matrix of the joint corresponding to the uncoupled degrees of freedom and $\mathbf{y}$ is the associated generalized coordinate vector. Condensation onto the interface degrees of freedom gives

$$
\left(\mathbf{D}_{\mathrm{ci}} \boldsymbol{\Psi}_{\mathrm{i}}\left(\boldsymbol{\Psi}_{\mathrm{i}}^{\mathrm{T}} \mathbf{D}_{\mathrm{ii}} \boldsymbol{\Psi}_{\mathrm{i}}\right)^{-1} \boldsymbol{\Psi}_{\mathrm{i}}^{\mathrm{T}} \mathbf{D}_{\mathrm{ic}}-\mathbf{D}_{\mathrm{cc}}\right) \overline{\mathbf{q}}_{\mathrm{c}}-\mathbf{D}_{\mathrm{ci}} \boldsymbol{\Psi}_{\mathrm{i}}\left(\boldsymbol{\Psi}_{\mathrm{i}}^{\mathrm{T}} \mathbf{D}_{\mathrm{ii}} \boldsymbol{\Psi}_{\mathrm{i}}\right)^{-1} \boldsymbol{\Psi}_{\mathrm{i}}^{\mathrm{T}} \overline{\mathbf{f}}_{\mathrm{i}}=\overline{\mathbf{f}}_{\mathrm{c}}
$$

In contrast with Eq. (17), the inversion of $\mathbf{D}_{\mathrm{ii}}$ is transformed into the inversion of $\boldsymbol{\Psi}_{\mathrm{i}}^{\mathrm{T}} \mathbf{D}_{\mathrm{ii}} \boldsymbol{\Psi}_{\mathrm{i}}$ by using the modal expansion technique, i.e. 


$$
\mathbf{D}_{\mathrm{ii}}^{-1}=\boldsymbol{\Psi}_{\mathrm{i}}\left(\boldsymbol{\Psi}_{\mathrm{i}}^{\mathrm{T}} \mathbf{D}_{\mathrm{ii}} \boldsymbol{\Psi}_{\mathrm{i}}\right)^{-1} \boldsymbol{\Psi}_{\mathrm{i}}^{\mathrm{T}}
$$

which significantly reduces the computational load.

\subsection{Displacement-force relation of the plates at the coupling interfaces}

The displacements and internal forces at the location $\left(x_{\mathrm{r}}, y_{\mathrm{r}}\right)$ of a plate can be expressed in terms of waves as [43]

$$
\begin{aligned}
& \mathbf{z}\left(x_{\mathrm{r}}, y_{\mathrm{r}}\right)=\mathbf{A}\left(x_{\mathrm{r}}\right) \widehat{\mathbf{T}}\left(y_{\mathrm{r}}\right) \mathbf{a}+\mathbf{A}\left(x_{\mathrm{r}}\right) \mathbf{T}_{\mathrm{l}}\left(y_{\mathrm{r}}\right) \mathbf{e}, y_{\mathrm{r}} \leq y_{\mathrm{e}} \\
& \mathbf{z}\left(x_{\mathrm{r}}, y_{\mathrm{r}}\right)=\mathbf{A}\left(x_{\mathrm{r}}\right) \widehat{\mathbf{T}}\left(y_{\mathrm{r}}\right) \mathbf{a}+\mathbf{A}\left(x_{\mathrm{r}}\right) \mathbf{T}_{\mathrm{r}}\left(y_{\mathrm{r}}\right) \mathbf{e}, y_{\mathrm{r}}>y_{\mathrm{e}}
\end{aligned}
$$

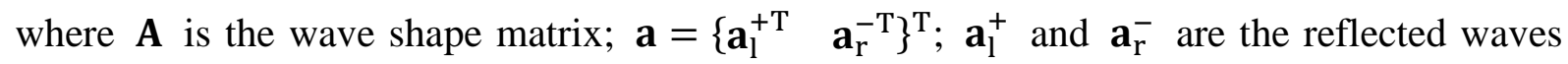
at the left and right ends of the plate, respectively; $\mathbf{e}$ is the directly excited wave; $y_{\mathrm{e}}$ is the coordinate of the excitation in the $y$ direction; and

$$
\begin{gathered}
\widehat{\mathbf{T}}(y)=\left[\begin{array}{cc}
\mathbf{T}(y) & \mathbf{0} \\
\mathbf{0} & \mathbf{T}(b-y)
\end{array}\right], \quad \mathbf{T}_{1}=\left[\begin{array}{ccc}
\mathbf{0} & \mathbf{0} \\
\mathbf{0} & \mathbf{T}\left(y_{\mathrm{e}}-y\right)
\end{array}\right], \quad \mathbf{T}_{\mathrm{r}}=\left[\begin{array}{ccc}
\mathbf{T}\left(y-y_{\mathrm{e}}\right) & \mathbf{0} \\
\mathbf{0} & \mathbf{0}
\end{array}\right] \\
\mathbf{T}(y)=\left[\begin{array}{cccc}
\mathrm{e}^{\mu_{y 1} y} & 0 & \cdots & 0 \\
0 & \mathrm{e}^{\mu_{y 2} y} & \cdots & 0 \\
\vdots & \vdots & \ddots & \vdots \\
0 & 0 & \cdots & \mathrm{e}^{\mu_{y m} y}
\end{array}\right]
\end{gathered}
$$

where the sub-matrix $\mathbf{0}$ has the same order as $\mathbf{T}$ and $m$ is the number of positively travelling waves. More details are given in [43].

For a plate whose left end is uncoupled, the displacement-force relation at the coupling edge can be expressed as

$$
\begin{aligned}
& \overline{\mathbf{q}}=\overline{\mathbf{A}} \widehat{\mathbf{T}}(b)\left\{\begin{array}{c}
\mathbf{R}_{\mathrm{l} 1} \\
\mathbf{I}
\end{array}\right\} \mathbf{P F} \\
& +\overline{\mathbf{A}} \widehat{\mathbf{T}}(b)\left\{\begin{array}{c}
\mathbf{R}_{\mathbf{l} 1} \\
\mathbf{I}
\end{array}\right\} \mathbf{P \Theta}\left(\widehat{\mathbf{T}}(b)\left\{\begin{array}{c}
\mathbf{R}_{\mathrm{l} 2} \\
\mathbf{0}
\end{array}\right\}+\mathbf{T}_{\mathrm{r}}(b) \mathbf{e}\right)+\overline{\mathbf{A}} \widehat{\mathbf{T}}(b)\left\{\begin{array}{c}
\mathbf{R}_{\mathrm{l} 2} \\
\mathbf{0}
\end{array}\right\}+\overline{\mathbf{A}} \mathbf{T}_{\mathrm{r}}(b) \mathbf{e}
\end{aligned}
$$


For a plate whose right end is uncoupled, the displacement-force relation at the coupling edge can be expressed as

$$
\begin{aligned}
& \overline{\mathbf{q}}=\overline{\mathbf{A}} \widehat{\mathbf{T}}(0)\left\{\begin{array}{c}
\mathbf{I} \\
\mathbf{R}_{\mathrm{r} 1}
\end{array}\right\} \mathbf{Q F} \\
& -\overline{\mathbf{A}} \widehat{\mathbf{T}}(0)\left\{\begin{array}{c}
\mathbf{I} \\
\mathbf{R}_{\mathrm{r} 1}
\end{array}\right\} \mathbf{Q \Theta}\left(\widehat{\mathbf{T}}(0)\left\{\begin{array}{c}
\mathbf{0} \\
\mathbf{R}_{\mathrm{r} 2}
\end{array}\right\}+\mathbf{T}_{1}(0) \mathbf{e}\right)+\overline{\mathbf{A}} \widehat{\mathbf{T}}(0)\left\{\begin{array}{c}
\mathbf{0} \\
\mathbf{R}_{\mathrm{r} 2}
\end{array}\right\}+\overline{\mathbf{A}} \mathbf{T}_{\mathrm{l}}(0) \mathbf{e}
\end{aligned}
$$

The detailed derivations of Eqs. $(24,25)$ are omitted here for simplicity and can be found in Appendix A.

\subsection{System solution formulation for the whole structure}

Section 4.2 gives the displacement-force relations at the coupling edges of each plate component, i.e., Eqs. (24) and (25). The combination of these with Eq. (17) establishes the solution framework for the whole structure, subject to compatibility of the coupling forces at the coupling edges of the plates and the joint. Discretizing the coupling edges according to the finite element mesh of the joint shown in Fig. 3, the wave relevant force vector can be calculated approximately as

$$
\begin{gathered}
\mathbf{F}=\int_{0}^{a} \mathbf{A}_{\mathbf{x}}(x) \mathbf{f}(x) \mathrm{d} x=\mathbf{A}_{\mathbf{x}}\left(x_{1}\right) \int_{0}^{\frac{\delta_{1}}{2}} \mathbf{f}(x) \mathrm{d} x \\
+\mathbf{A}_{\mathbf{x}}\left(x_{n}\right) \int_{x_{n}-\frac{\delta_{n-1}}{2}} \mathbf{f}(x) \mathrm{d} x+\sum_{i=2}^{n-1} \mathbf{A}_{\mathbf{x}}\left(x_{i}\right) \int_{x_{i}-\frac{\delta_{i-1}}{2}}^{x_{n}} \mathbf{f}(x) \mathrm{d} x
\end{gathered}
$$

where $\delta_{i}=x_{i+1}-x_{i}$, and $x_{i}(i=1, \ldots n)$ are the coordinates of the discrete nodes at the coupling edge of the joint. Additionally, the finite element relevant force vector can be calculated from

$$
\int_{0}^{\frac{\delta_{1}}{2}} \mathbf{f}(x) \mathrm{d} x=\overline{\mathbf{f}}_{1}, \quad \int_{x_{n}-\frac{\delta_{n-1}}{2}}^{x_{n}} \mathbf{f}(x) \mathrm{d} x=\overline{\mathbf{f}}_{n}, \quad \int_{x_{i}-\frac{\delta_{i-1}}{2}}^{x_{i}+\frac{\delta_{i}}{2}} \mathbf{f}(x) \mathrm{d} x=\overline{\mathbf{f}}_{i}, i=2,3, \ldots, n-1
$$


Now Eq. (27) can be written as

$$
\mathbf{F}=\overline{\mathbf{A}}_{\mathbf{x}} \overline{\mathbf{f}}
$$

where $\overline{\mathbf{f}}=\left[\begin{array}{llll}\overline{\mathbf{f}}_{1}^{\mathrm{T}} & \overline{\mathbf{f}}_{2}^{\mathrm{T}} & \cdots & \overline{\mathbf{f}}_{n}^{\mathrm{T}}\end{array}\right]^{\mathrm{T}}$. For flexural and in-plane vibration,

$\overline{\mathbf{A}}_{\mathbf{x}}=\left[\begin{array}{lllllll}\overline{\mathbf{A}}_{w}^{+\mathrm{T}}\left(x_{1}\right) & \overline{\mathbf{A}}_{\theta}^{+\mathrm{T}}\left(x_{1}\right) & \overline{\mathbf{A}}_{w}^{+\mathrm{T}}\left(x_{2}\right) & \overline{\mathbf{A}}_{\theta}^{+\mathrm{T}}\left(x_{2}\right) & \cdots & \overline{\mathbf{A}}_{w}^{+\mathrm{T}}\left(x_{n}\right) & \overline{\mathbf{A}}_{\theta}^{+\mathrm{T}}\left(x_{n}\right)\end{array}\right]^{\mathrm{T}}$ and

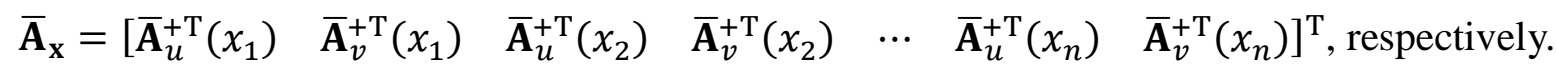

Eqs. (24), (25) and (28) are all based on local coordinates. For convenience, the in-plane variables and flexural variables are put together, so that on the coupling interface of the $i$ th plate

$$
\overline{\mathbf{q}}_{i}=\left\{\begin{array}{c}
\overline{\mathbf{q}}_{i, \text { in }} \\
\overline{\mathbf{q}}_{i, \text { out }}
\end{array}\right\}, \quad \overline{\mathbf{f}}_{i}=\left\{\begin{array}{c}
\overline{\mathbf{f}}_{i, \text { in }} \\
\overline{\mathbf{f}}_{i, \text { out }}
\end{array}\right\}
$$

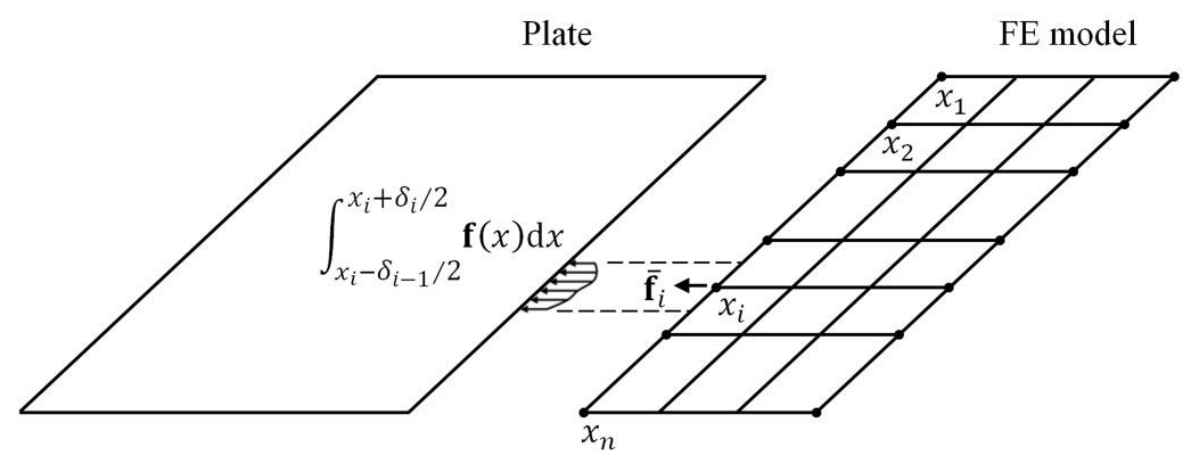

Fig. 3. Interconnection between plates and joints

By combining Eqs. (24), (25) and (28), assembling the equations of each plate gives

$$
\overline{\mathbf{q}}_{\mathrm{c}}=\mathbf{H} \overline{\mathbf{f}}_{\mathrm{c}}+\mathbf{G}
$$

where $\mathbf{H}=\operatorname{diag}\left\{\mathbf{h}_{1}, \mathbf{h}_{2}, \ldots, \mathbf{h}_{N}\right\}, \mathbf{G}=\left[\mathbf{g}_{1}^{\mathrm{T}}, \mathbf{g}_{2}^{\mathrm{T}}, \ldots, \mathbf{g}_{N}^{\mathrm{T}}\right]^{\mathrm{T}}$, and the detailed derivations are given in Appendix B.

Combining Eqs. (30) and (17) gives 


$$
\overline{\mathbf{f}}_{\mathrm{c}}=(\mathbf{I}-\boldsymbol{\alpha} \mathbf{H})^{-1}(\boldsymbol{\alpha} \mathbf{G}+\boldsymbol{\beta})
$$

The wave amplitudes of each plate can be obtained by combining Eqs. (A.6) and (A.9) and the reflections at the uncoupled ends of each plate. The input mobility and the input power associated with each wave can also be obtained (the formulation of the input power is presented in Appendix C, and more details can be found in [44]), while the response of each plate can be evaluated from Eq. (21).

\subsection{Wave scattering at the joint}

The wave scattering matrix $\mathbf{S}$ can be obtained for the hybrid solution formulation by applying plane wave excitation of each wave component in turn, assuming the length of each plate to be semi-infinite. This assumption is equivalent to setting the reflection coefficient at the uncoupled end of each plate to zero which gives $\mathbf{R}_{11}=\mathbf{0}, \mathbf{R}_{\mathrm{r} 1}=\mathbf{0}$. Once $\mathbf{S}$ is obtained, the finite element model of the structural joint can be replaced by $\mathbf{S}$ in the hybrid solution in a new analysis. As shown in Fig. 1, the wave scattering relation provided by the joint has

$$
\hat{\mathbf{a}}=\mathbf{S} \tilde{\mathbf{a}}
$$

where $\hat{\mathbf{a}}=\left[\begin{array}{llllll}\mathbf{a}_{1 \mathrm{r}}^{-\mathrm{T}} & \mathbf{a}_{2 \mathrm{l}}^{+\mathrm{T}} & \ldots & \mathbf{a}_{i \mathrm{l}}^{+\mathrm{T}} & \ldots & \mathbf{a}_{N \mathrm{l}}^{+\mathrm{T}}\end{array}\right]^{\mathrm{T}}, \tilde{\mathbf{a}}=\left[\begin{array}{llllll}\mathbf{a}_{1 \mathrm{r}}^{+\mathrm{T}} & \mathbf{a}_{2 \mathrm{l}}^{-\mathrm{T}} & \ldots & \mathbf{a}_{i \mathrm{l}}^{-\mathrm{T}} & \ldots & \mathbf{a}_{N \mathrm{l}}^{-\mathrm{T}}\end{array}\right]^{\mathrm{T}}$.

Combining Eqs. (A.3) and (A.8) gives

$$
\tilde{\mathbf{a}}=\widetilde{\mathbf{R}} \hat{\mathbf{a}}+\tilde{\mathbf{e}}
$$

where $\widetilde{\mathbf{R}}=\operatorname{diag}\left\{\mathbf{T}(b) \mathbf{R}_{1,11}, \mathbf{T}(b) \mathbf{R}_{2, \mathrm{r} 1}, \cdots, \mathbf{T}(b) \mathbf{R}_{i, \mathrm{r} 1}, \cdots, \mathbf{T}(b) \mathbf{R}_{N, \mathrm{r} 1}\right\}$, 


$$
\tilde{\mathbf{e}}=\left\{\begin{array}{c}
\mathbf{T}\left(b-y_{\mathrm{e}}\right) \mathbf{e}_{1}^{+}+\mathbf{T}(b) \mathbf{R}_{1,11} \mathbf{T}\left(y_{\mathrm{e}}-b\right) \mathbf{e}_{1}^{-} \\
\mathbf{T}(b) \mathbf{R}_{2, \mathrm{r} 1} \mathbf{T}\left(-y_{\mathrm{e}}\right) \mathbf{e}_{2}^{+}+\mathbf{T}\left(y_{\mathrm{e}}\right) \mathbf{e}_{2}^{-} \\
\vdots \\
\mathbf{T}(b) \mathbf{R}_{i, \mathrm{r} 1} \mathbf{T}\left(-y_{\mathrm{e}}\right) \mathbf{e}_{i}^{+}+\mathbf{T}\left(y_{\mathrm{e}}\right) \mathbf{e}_{i}^{-} \\
\vdots \\
\mathbf{T}(b) \mathbf{R}_{N, \mathrm{r} 1} \mathbf{T}\left(-y_{\mathrm{e}}\right) \mathbf{e}_{N}^{+}+\mathbf{T}\left(y_{\mathrm{e}}\right) \mathbf{e}_{N}^{-}
\end{array}\right\}
$$

The wave amplitudes can be obtained from Eqs. (32) and (33) as

$$
\hat{\mathbf{a}}=(\mathbf{I}-\mathbf{S} \widetilde{\mathbf{R}})^{-1} \mathbf{S} \widetilde{\mathbf{e}}
$$

Similarly, the input power can be obtained from the amplitude associated with each wave, and meanwhile the response of each plate can be obtained from Eq. (21).

In this section, the input power and the scattering coefficient associated with each wave subsystem are obtained through a hybrid analytical wave and finite element analysis. The energy distribution of the built-up structure can now be evaluated by solving Eq. (13).

\section{Numerical examples}

\subsection{Validation of the hybrid solution formulation}

\subsubsection{Three angle coupled plates with a joint}

The effectiveness of the hybrid analytical wave and finite element formulation is now validated. Consider a built-up structure composed of three rectangular plates coupled through a structural joint, with angles of $120^{\circ}$ between adjacent plates, as shown in Fig. 4 . The three plates have the same geometry and material properties, namely length $b=0.2 \mathrm{~m}$, width $a=0.1 \mathrm{~m}$, thickness $h=0.001 \mathrm{~m}$, Young's modulus $E=200 \mathrm{GPa}$, density $\rho=7800 \mathrm{~kg} / \mathrm{m}^{3}$ and Poisson's ratio $v=0.3$. All plates are simply supported along $x=0, a$, the in-plane boundary condition is SS1-SS2 [46], and all the uncoupled ends of the plates are free. The 
material properties of the joint are the same as those of the plate components. The width and thickness of the joint are $0.005 \mathrm{~m}$ and $0.002 \mathrm{~m}$, respectively, and the two ends of the joint are clamped. An external unit force $f^{\text {ext }}$ is applied normally at the location $(0.4 a, 0.6 b)$ of the first plate.

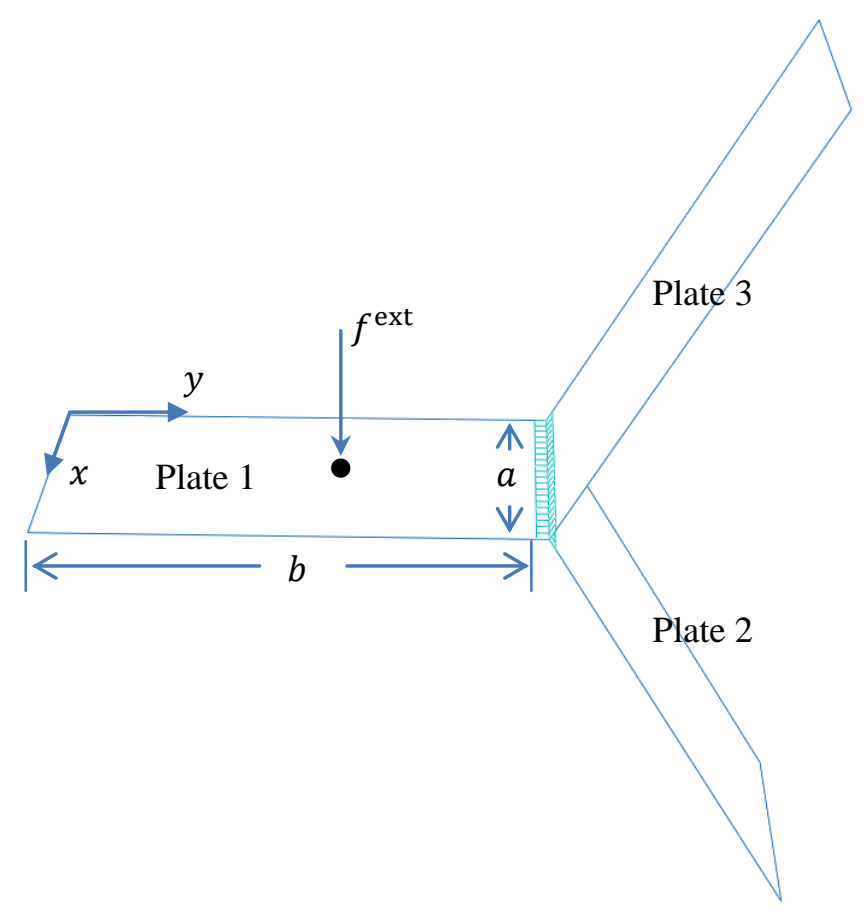

Fig. 4. Three angle coupled plates with a joint

In the hybrid formulation, the finite element model of the joint is constructed using the commercial software ABAQUS [47] with shell element S4R and element size $0.0025 \mathrm{~m}$ according to which the joint is divided into 240 elements. The complete finite analysis which is used to validate the presented hybrid solution formulation is also performed using ABAQUS. Four types of complete finite element analysis are given, with element sizes 0.001 $\mathrm{m}$ and $0.0025 \mathrm{~m}$ (discretizing the structure into 61500 and 9840 elements, respectively), and 
truncated at 1000 and 500 modes for each element size. The complete finite element result with element size $0.001 \mathrm{~m}$ and 1000 modes is considered as the reference result. Fig. 5 gives the absolute value of the relative error in the magnitude of the input mobilities calculated by the hybrid formulation and the other three complete finite element analyses with respect to the reference result. The result has 60 waves for each plate, and is truncated at 40 modes for the joint using the modal expansion technique. It can be seen from Fig. 5 that the accuracy of the present method is better than that of the complete finite analysis with element size $0.0025 \mathrm{~m}$ over a large range, especially at higher frequencies, and is comparable to that of complete finite element analysis with element size $0.001 \mathrm{~m}$ and 500 modes. The corresponding input mobilities are given in Fig. 6. The CPU time of the presented hybrid analysis and the complete finite element analysis are $448 \mathrm{~s}$ and $1178 \mathrm{~s}$, respectively. Hence the hybrid formulation has high accuracy and efficiency.

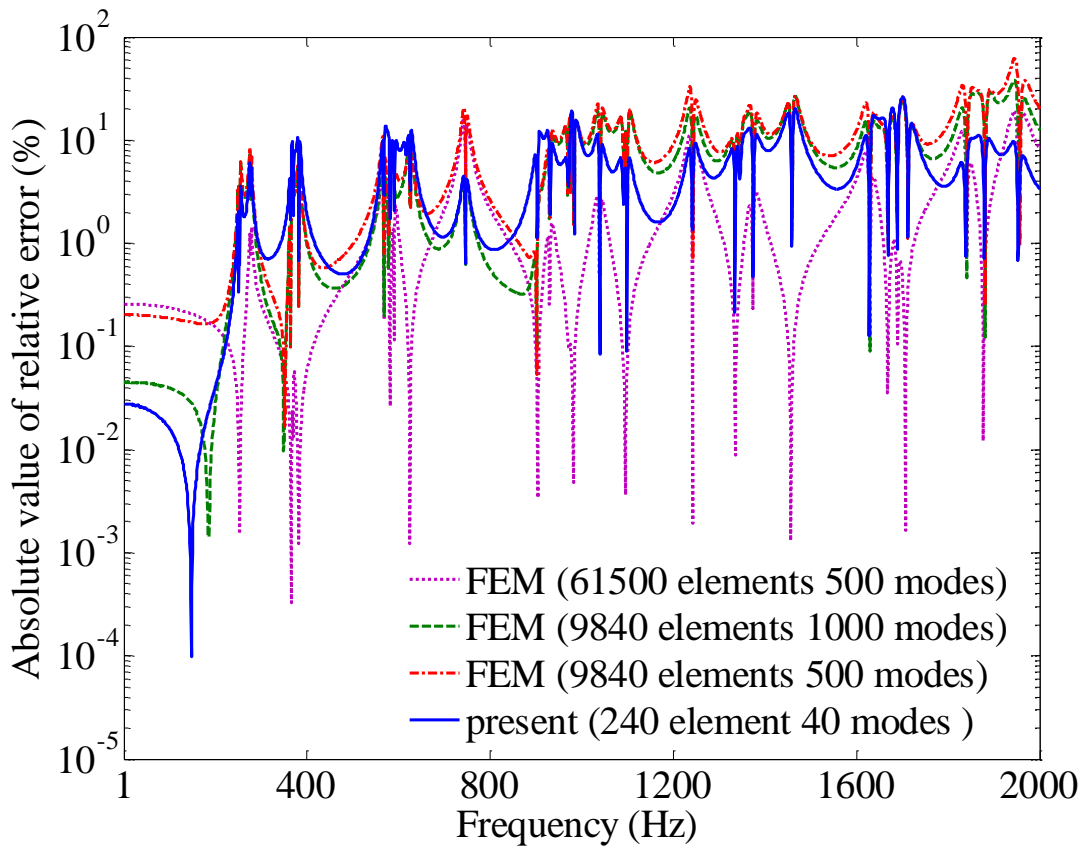


Fig. 5. Absolute value of relative error of the magnitude of the input mobilities calculated by the hybrid formulation and complete finite element analysis with respect to the reference result

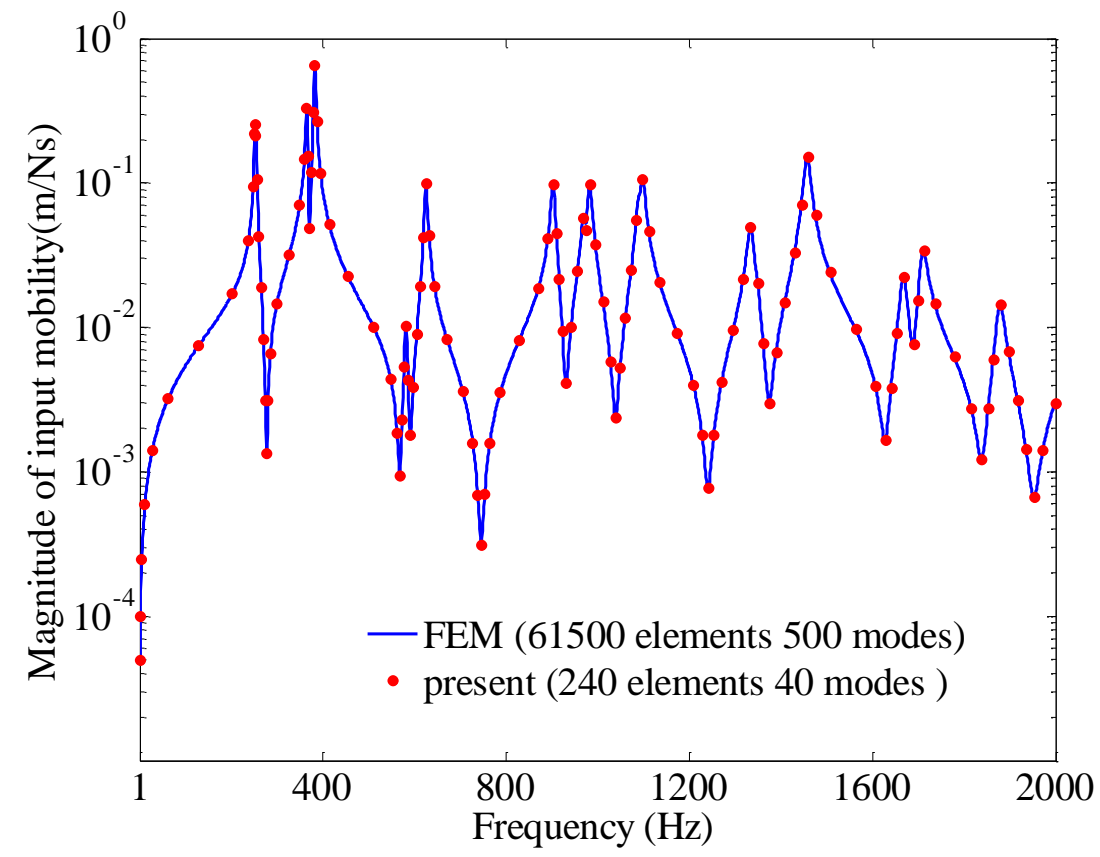

Fig. 6. Magnitude of the input mobilities calculated by the hybrid formulation and complete finite element analysis

A feature of the hybrid formulation is that the computational time decreases significantly with increasing length of the plates. The contribution of the near field waves of high order attenuates rapidly with the propagation distance, and so fewer waves are needed to obtain an accurate result as the plate length/width ratio increases. In contrast, the complete finite element analysis needs more elements to ensure the accuracy of the result, increasing its computational time significantly. Fig. 7 gives the results of the same analysis as that of Fig. 5, with the length of each plate increased from $0.2 \mathrm{~m}$ to $0.6 \mathrm{~m} .181500$ and 29040 elements are now needed for the complete finite element models with element size $0.001 \mathrm{~m}$ and $0.0025 \mathrm{~m}$, 
respectively. The hybrid solution is based on 40 waves for each plate component, and the error level is significantly less than that of the other three complete finite element analyses over a great part of the frequency range, especially at high frequencies. Fig. 8 gives the magnitude of the input mobilities from the hybrid solution formulation and the complete finite element analysis with element size $0.001 \mathrm{~m}$ and 500 modes, and the corresponding CPU times are 333 s and 2761 s, respectively. Thus, with increasing plate length, the computational time of the hybrid solution reduces significantly, while that of the complete finite element analysis increases significantly.

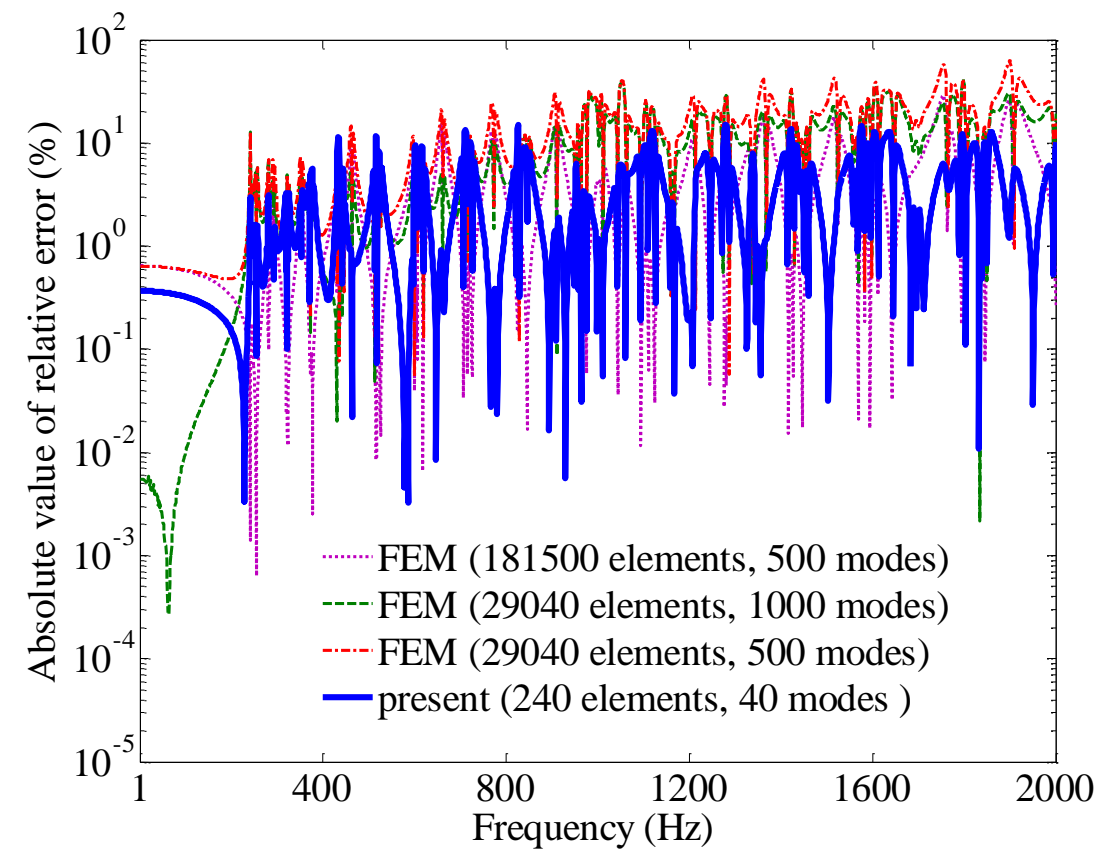

Fig. 7. Absolute value of relative error of the magnitude of the input mobilities calculated by the hybrid formulation and complete finite element analysis with respect to the reference result 


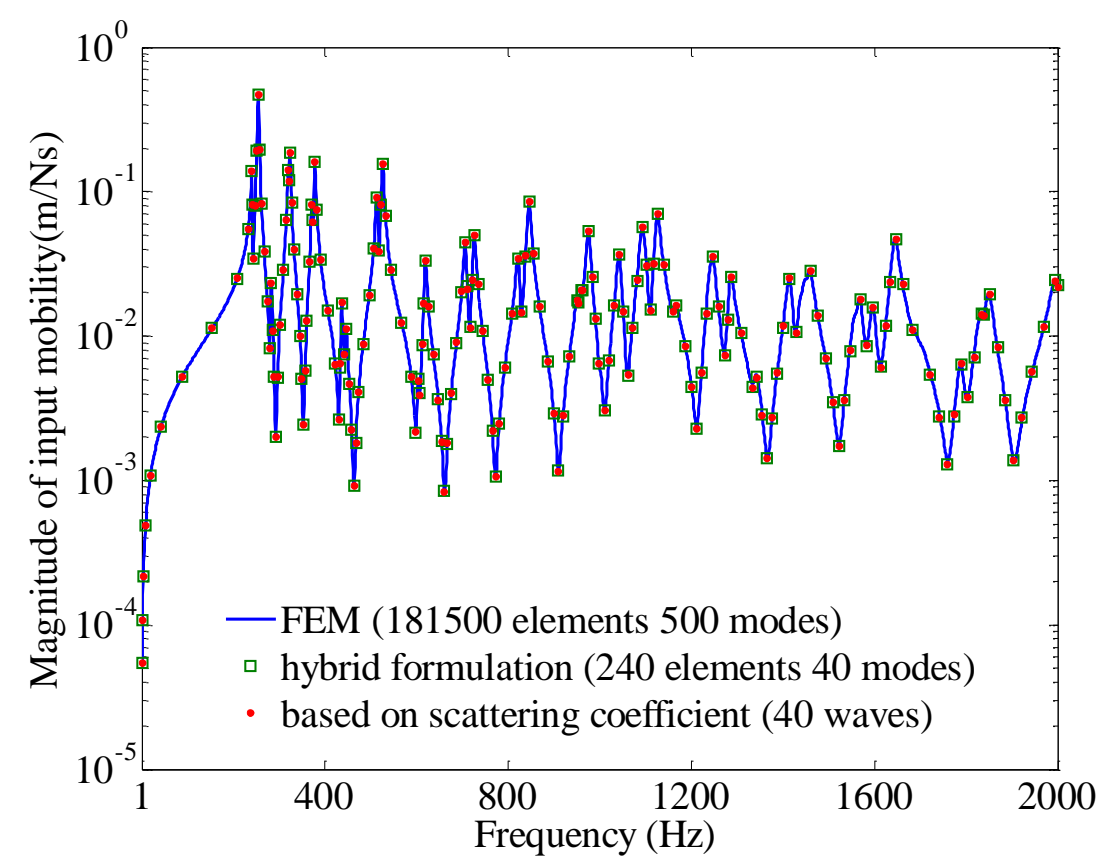

Fig. 8. Magnitude of the input mobilities from the hybrid formulation, complete finite element analysis and the model based on scattering coefficient

In addition, the wave scattering property of the joint obtained by the hybrid formulation further significantly enhances the computational efficiency. In a new analysis the finite element model of the joint in a hybrid solution can be replaced by the scattering coefficient, saving much computational time especially when the response of the built-up structure needs to be investigated under various forms of external excitation or for different plate lengths. The input mobility calculated based on this concept is also given in Fig. 8 and shows good agreement with the results from the complete hybrid solution and the complete finite element analysis, with a CPU time of just $25 \mathrm{~s}$ which reflects the significant advantage of this approach. 


\subsubsection{Efficiency of the modal expansion for the joint}

As shown in the previous example, the computational time of the hybrid formulation reduces significantly with increasing length/width ratio of the plates. When the joint is more rigid than the plates and the fundamental natural frequency of the joint is much larger than the upper limit of the analysis frequency range, a rather coarse finite element mesh for the joint is sufficient to give results of high accuracy. Consider the two plate structure shown in Fig. 9, where each plate has length $b=0.295 \mathrm{~m}$ and width $a=0.1 \mathrm{~m}$. An external unit force $f^{\text {ext }}$ is applied normally at the location $(0.4 a, 0.4 b)$ of the first plate. The length of the joint is 0.1 $\mathrm{m}$ and the cross section of the joint is a square of side $0.01 \mathrm{~m}$. Both ends of the joint are clamped. The material properties of all the components are the same as those of the previous example. For the complete finite element analysis, element size is $0.001 \mathrm{~m}$ (so 69000 elements are required), and 500 modes are used. For the hybrid formulation, two finite element meshes with 20 and 80 elements for the joint are adopted and the ABAQUS solid element C3D8R is used.

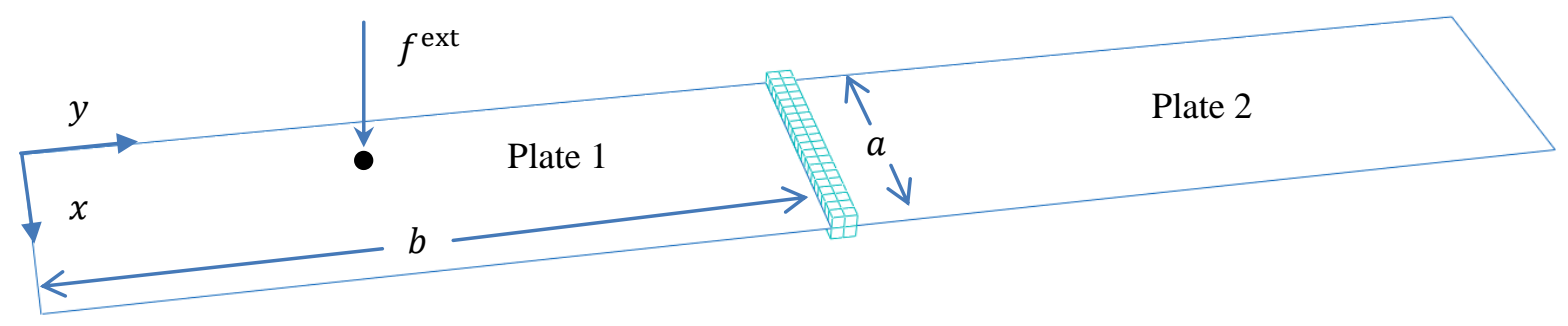

Fig. 9. Two co-planar coupled plates with a joint

Fig. 10 gives the absolute value of the relative error in the magnitude of the input mobilities from the hybrid solution with different numbers of modes for the joint, with respect 
to the hybrid solution without modal expansion, with 40 waves for each plate and 20 elements for the joint. It is seen that the relative error of the hybrid solution with 20 modes for the joint is very small. The CPU time is $16 \mathrm{~s}$, whereas that of the hybrid solution without modal expansion is 21 s. Fig. 11 gives the same analysis, but with 80 elements for the joint. Again the result with 20 modes for the joint has very good accuracy and the CPU time is $19 \mathrm{~s}$, compared with $50 \mathrm{~s}$ for the hybrid solution without modal expansion. The computational advantage of the modal expansion technique is obvious and increases with the number of elements in the joint. Fig. 12 gives the magnitudes of the input mobilities from the hybrid solution and the complete finite element analysis, where the hybrid solution is based on two meshes with 40 waves for each plate and 20 modes for the joint. The CPU time of the complete finite analysis is $2105 \mathrm{~s}$. As can be seen, the three results are in good agreement, and the effectiveness of the hybrid formulation and the modal expansion technique are validated.

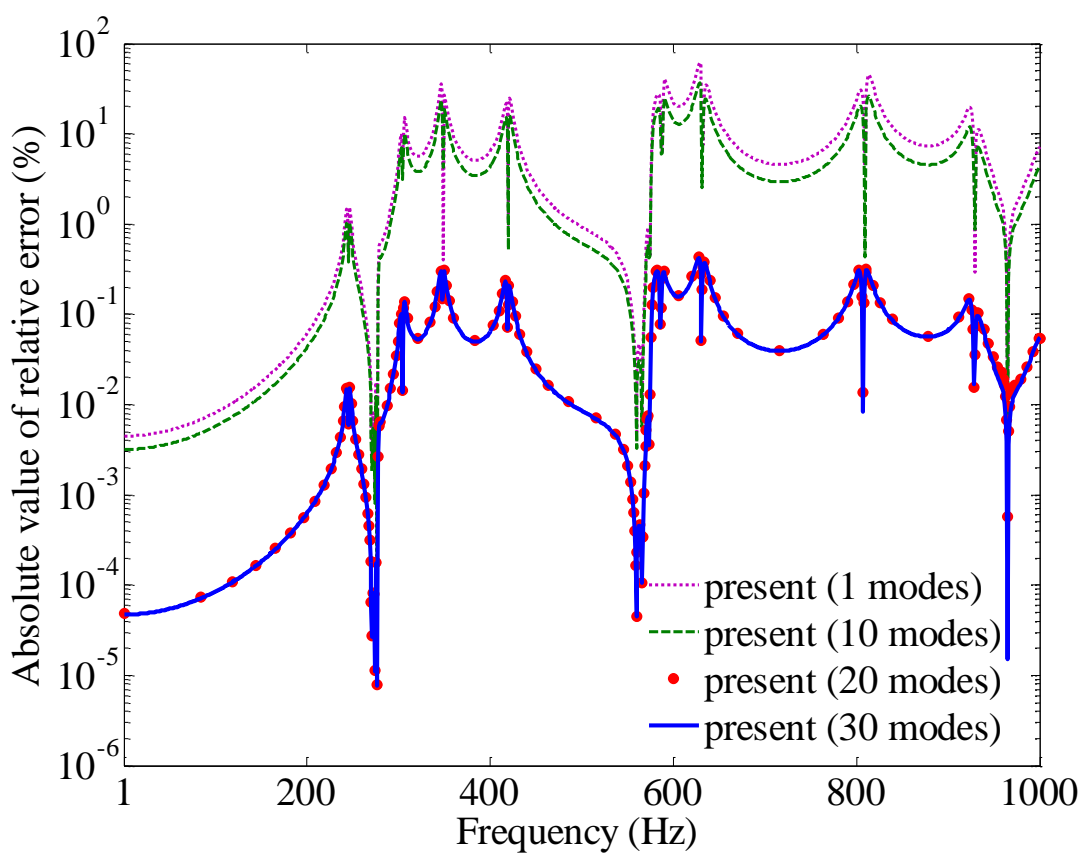

Fig. 10. Absolute value of relative error of magnitude of the input mobilities from the hybrid solution with different numbers of modes for the joint, with respect to the hybrid solution 
without modal expansion, with 40 waves for each plate and 20 elements for the joint.

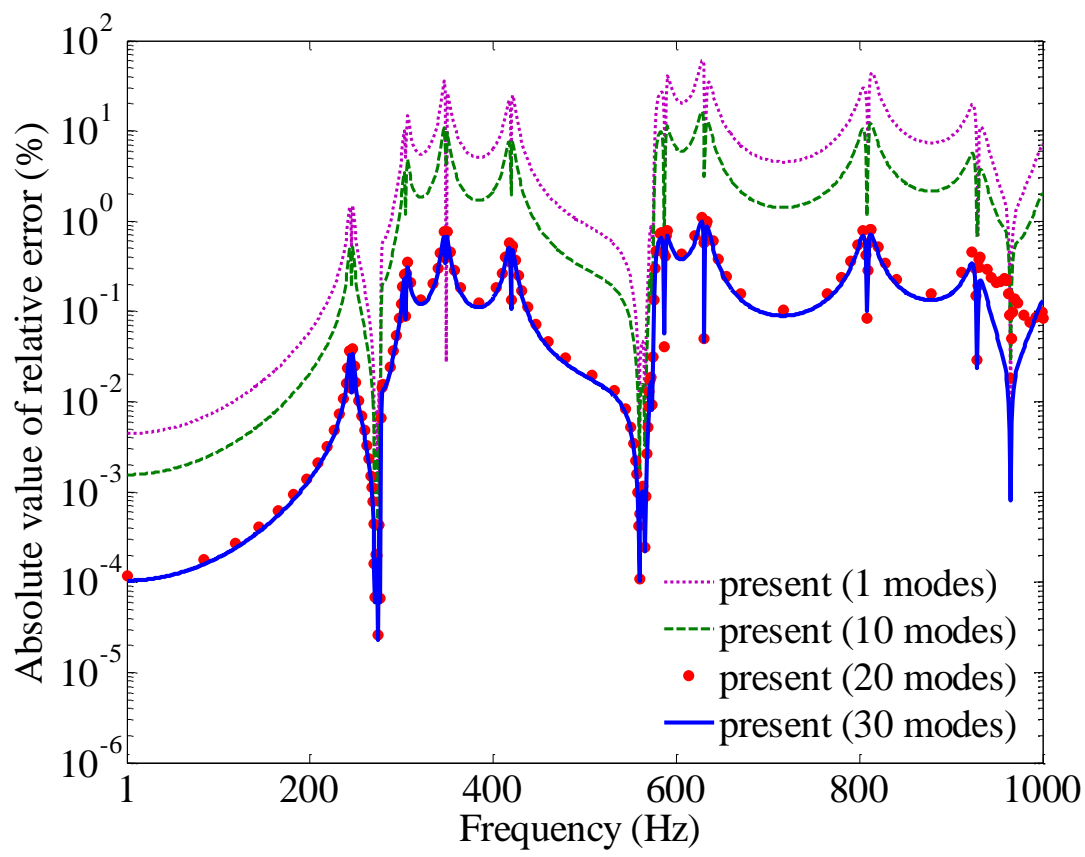

Fig. 11. Absolute value of relative error of magnitude of the input mobilities from the hybrid solution with different numbers of modes for the joint, with respect to the hybrid solution without modal expansion, with 40 waves for each plate and 80 elements for the joint.

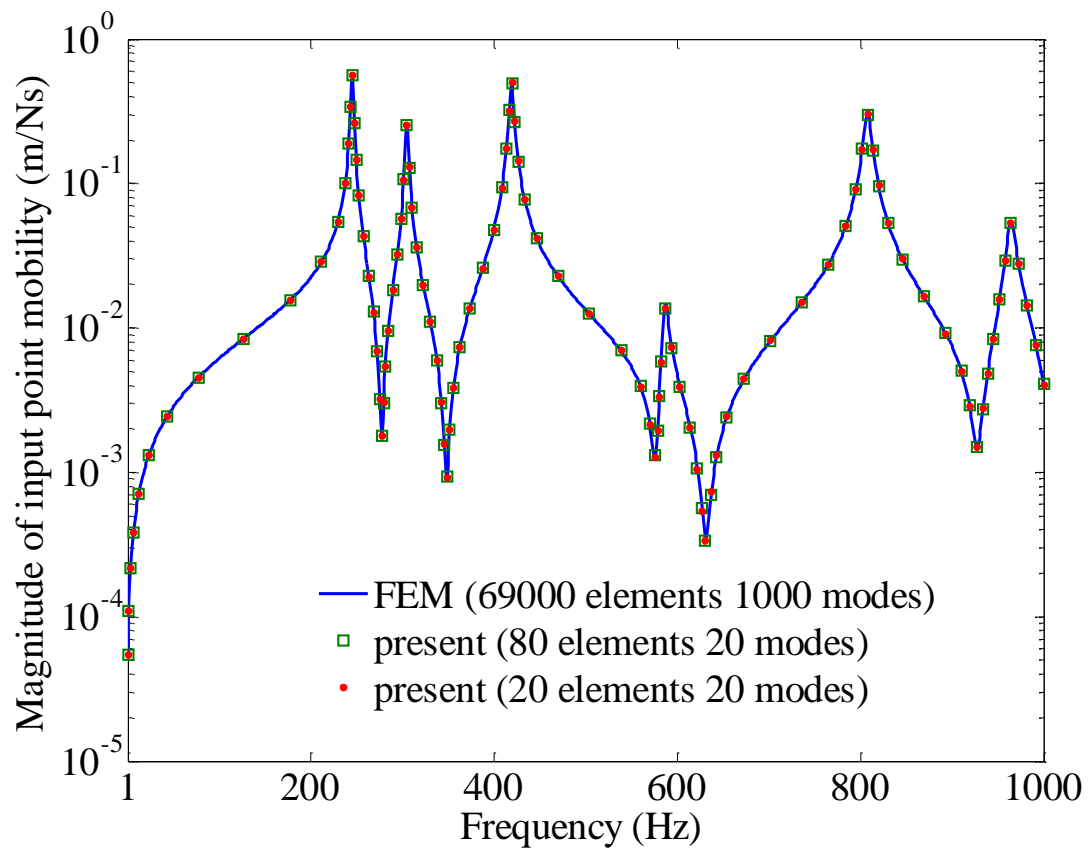

Fig. 12. Magnitudes of input mobilities from the hybrid solution and complete finite element 
analysis, where the hybrid solution is based on two meshes with 40 waves for each plate and 20 modes for the joint.

\subsection{Mode count and modal density}

In this section, the symplectic solutions of the mode count and the modal density are validated for a single rectangular plate of width $b=0.8 \mathrm{~m}$ and length $a=0.1 \mathrm{~m}$. The material properties of the plate are the same as those of the previous examples. The edges $x=0, a$ are simply supported and clamped respectively, i.e. SC. The present symplectic results are compared with FEM results and the classical asymptotic solution, where the FEM results are obtained using ABAQUS with S4R shell elements of size $0.002 \mathrm{~m}$. The classical asymptotic solution of the mode count is

$$
N(k)=\frac{A k^{2}}{4 \pi}
$$

Fig. 13 gives the mode counts calculated by the three methods for a rectangular plate with two ends simply supported. The present result is in good agreement with the FEM result, especially below $1219 \mathrm{~Hz}$. At this point the slope of the curve has a sudden dramatic jump because the second flexural wave becomes propagative. Fig. 14 gives the mode counts associated with three other boundary conditions, i.e., the two ends of the plate are both simply supported (SCSS), clamped (SCCC) and free (SCFF). The relationship between the results confirms Eq. (2). It can be seen from both these figures that the asymptotic solution is very different from the symplectic and FEM results. This is because the asymptotic solution is based on the assumption of high frequency vibration, whereas the structure studied here 
behaves with obvious mid-frequency characteristics, i.e. the modal information is significantly affected by the boundary conditions.

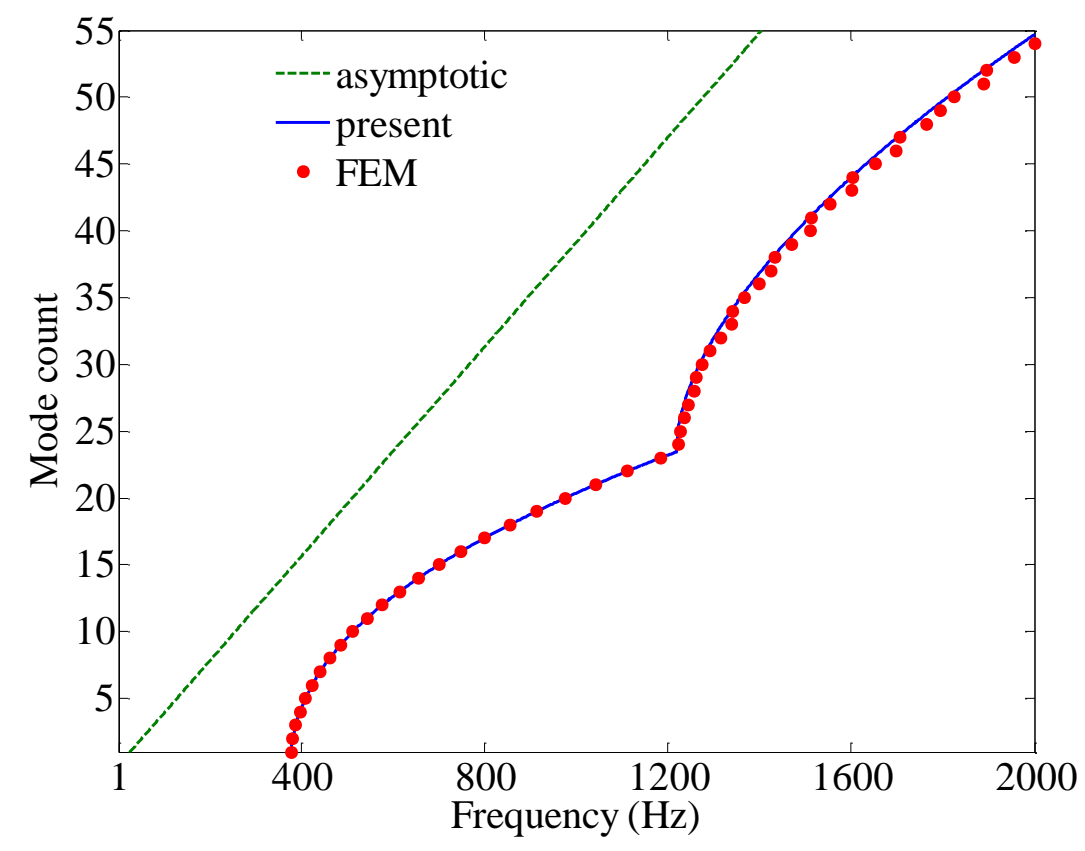

Fig. 13. Mode count from the present method, FEM and the asymptotic solution

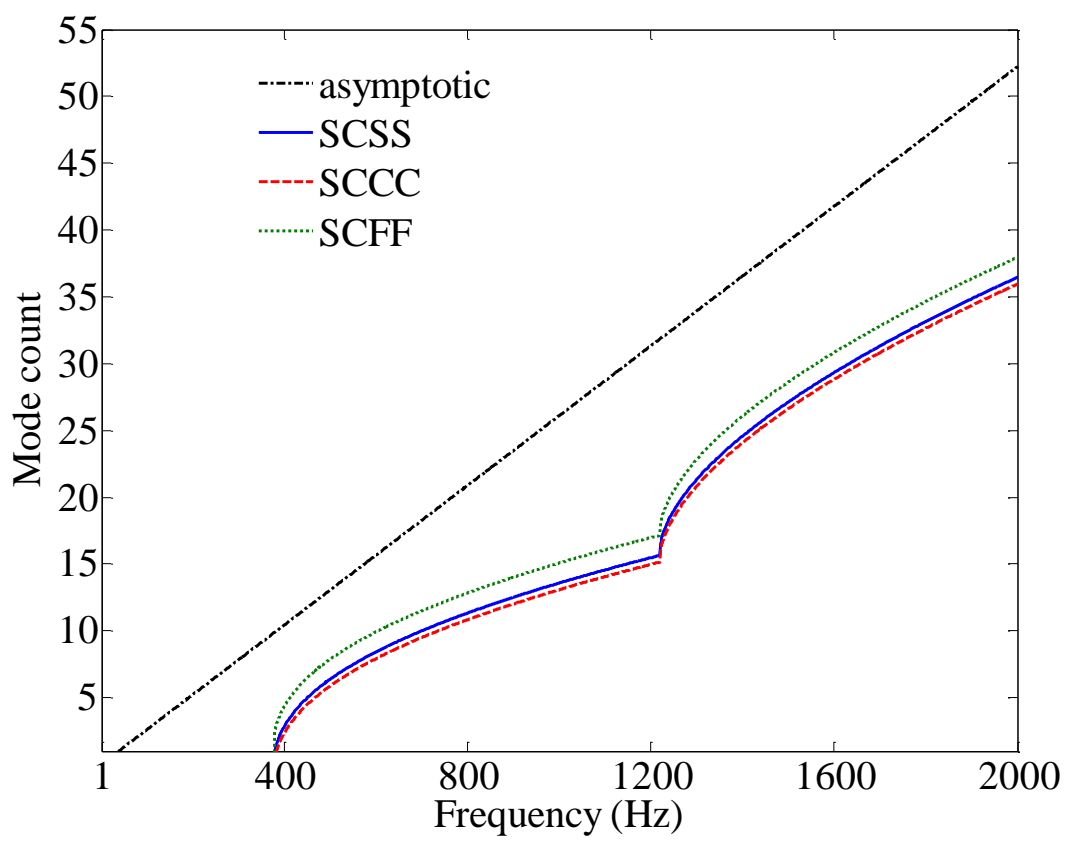

Fig. 14. Mode count from the present method with three different boundary conditions at the 
plate ends, and from the asymptotic solution

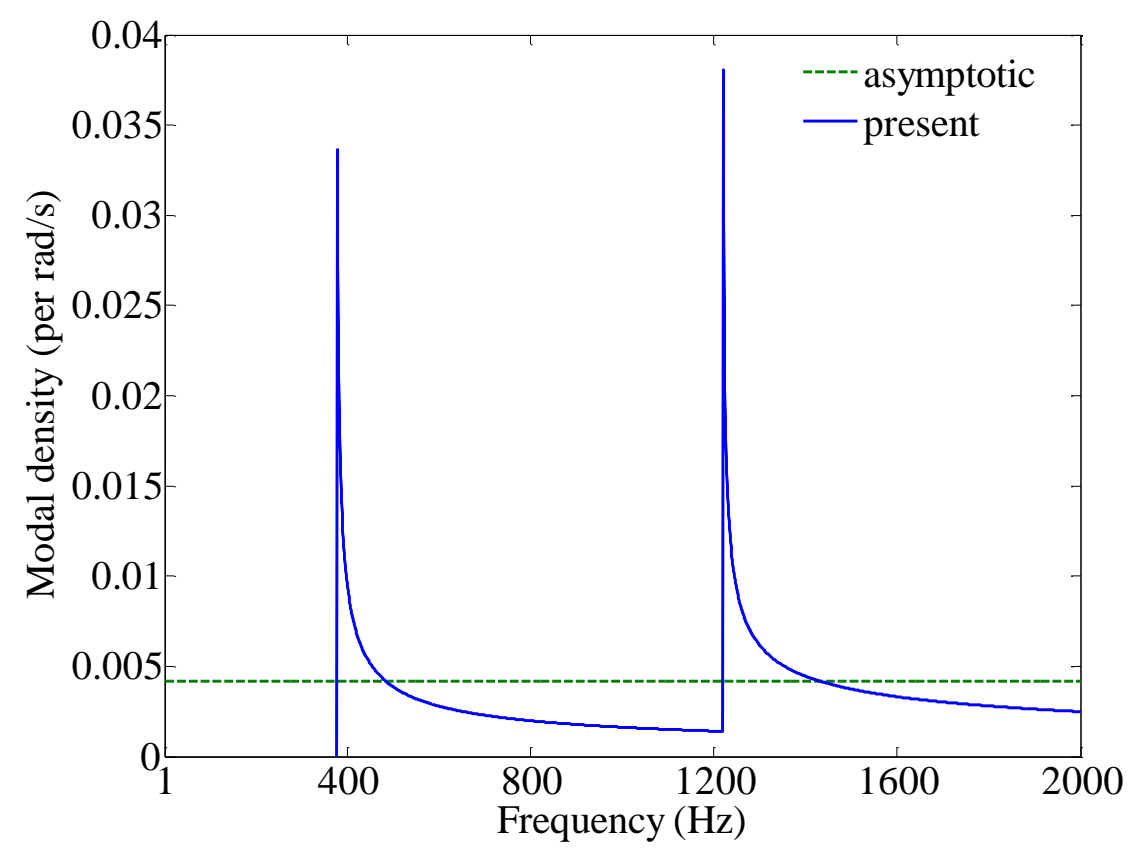

Fig. 15. Modal density from the present method and the asymptotic solution

Fig. 15 gives the modal densities of the plate flexural vibration from Eq. (6) and from the asymptotic solution $n(\omega)=\frac{A \omega}{2 \pi c_{\phi} c_{\mathrm{g}}}[3]$, where $c_{\phi}$ and $c_{\mathrm{g}}$ are the phase velocity and group velocity of the plate flexural wave field, respectively. As can be seen, the present result has obvious peak values at $377 \mathrm{~Hz}$ and $1219 \mathrm{~Hz}$. This is because the first and the second flexural waves respectively become propagative at these two frequencies, and the sudden jump from a zero mode count gives a significant peak value of the modal density. The peaks can be eliminated by averaging the results in the associated frequency bands. Fig. 16 presents modal densities averaged over one-third octave frequency bands, including the asymptotic solution, the present solution and the solution from Eq. (8) with the mode count calculated by the 
present method and FEM. The FEM result is an average of the three results for simply supported, clamped and free boundary conditions at the plate ends. Good agreement between the present result and the FEM result can be observed, and these results fluctuate strongly around the asymptotic solution. Fig. 17 gives the modal densities of the plate from the asymptotic solution and the present solution with three boundary conditions along the two opposite edges, i.e. simply supported and clamped (SC), simply supported (SS) and clamped (CC). It is seen that the boundary condition has a great influence on the modal density of the plate.

\subsection{Effectiveness of the presented energy flow analysis}

\subsubsection{Two co-planar plates coupled with a joint}

Consider a built-up structure comprising two co-planar plates coupled through a joint. For simplicity, the geometry and material properties of the two plates are the same as those of the example in section 5.2. The two opposite edges $x=0, a$ of each plate are both simply supported, and the uncoupled plate ends are free. The joint has width $0.02 \mathrm{~m}$, length $0.1 \mathrm{~m}$ and thickness $0.002 \mathrm{~m}$, and the same material properties as those of the plates. The two ends of the joint are both simply supported. Vertical deformation of the joint is prevented along the line joining the points with coordinates $(0.05 \mathrm{~m}, 0.005 \mathrm{~m})$ and $(0.05 \mathrm{~m}, 0.01 \mathrm{~m})$ with respect to the top left corner of the joint. An external unit force is applied normally at the location $(0.4 a, 0.4 b)$ of the first plate and the in-plane vibration of the plates is not considered. The mesh size of the joint in the present solution is $0.0025 \mathrm{~m}$. The complete finite element analysis is given by ABAQUS using S4R shell elements of size $0.0025 \mathrm{~m}$ and truncated at 500 
modes.

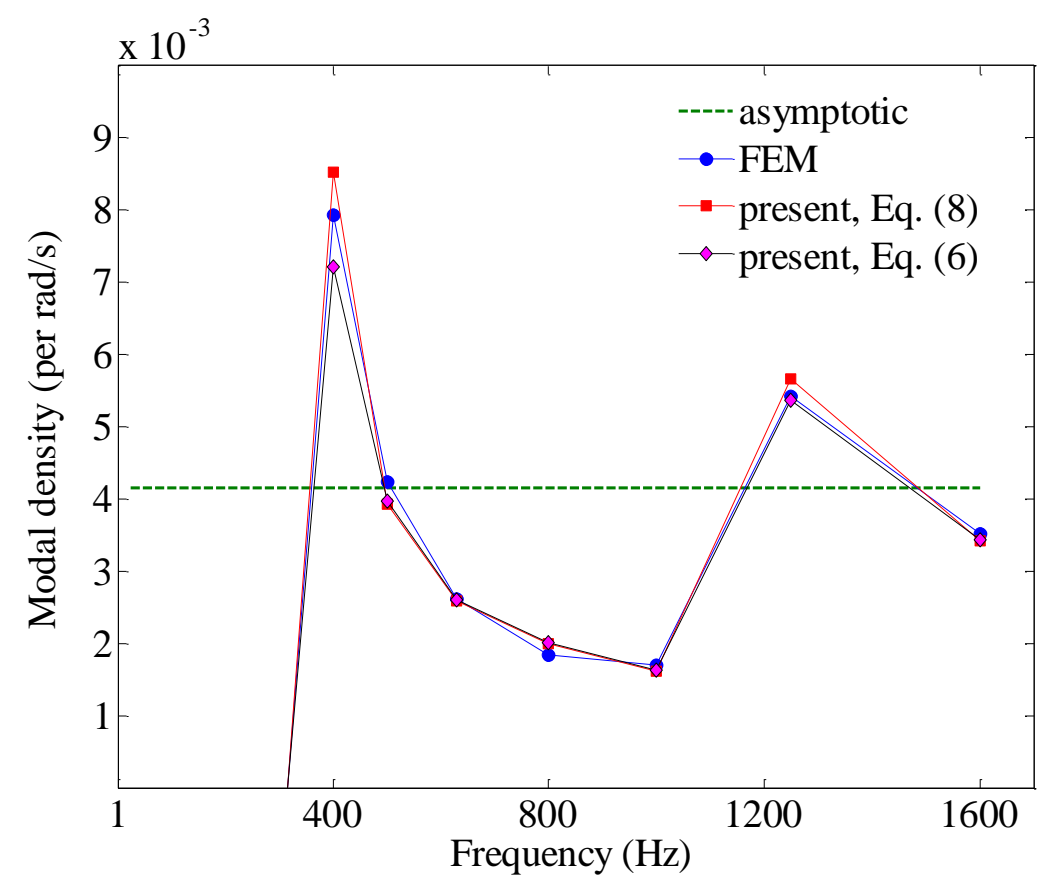

Fig. 16. Modal densities averaged over one-third octave frequency bands, including the asymptotic solution, the present solution and the solution from Eq. (8) with the mode count calculated by the present method and FEM.

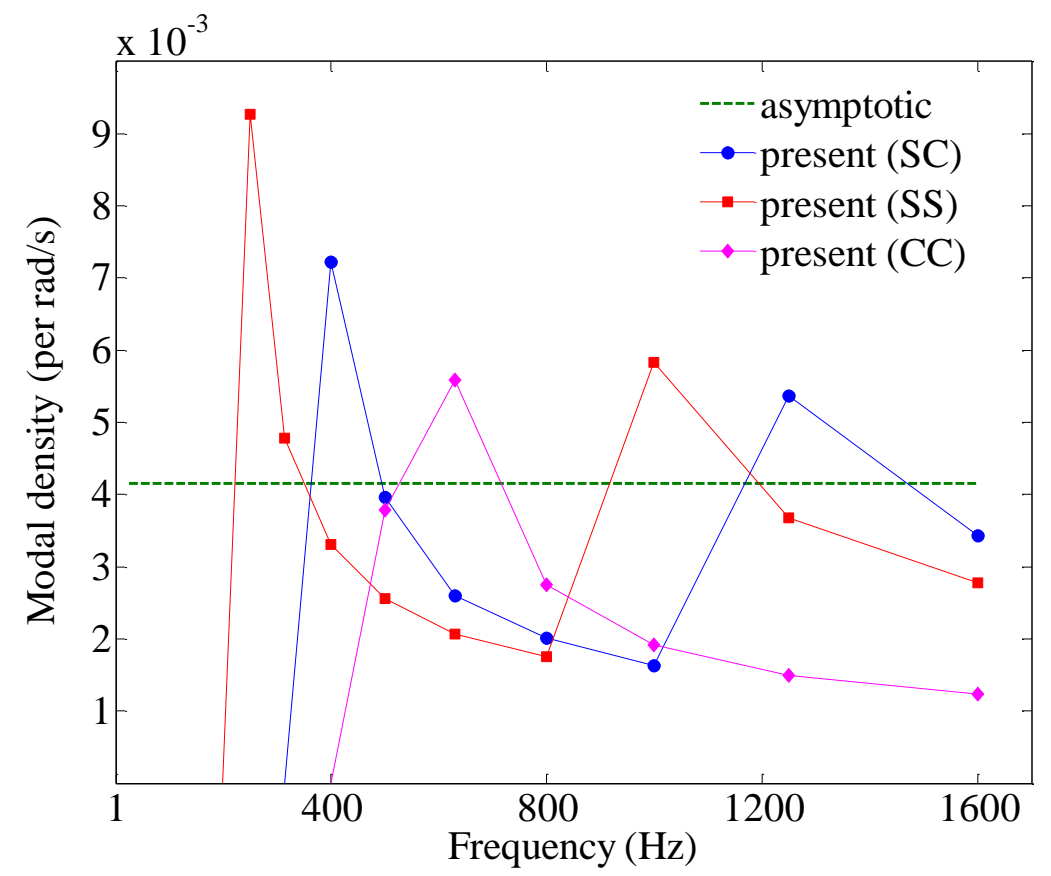

Fig. 17. Modal densities of the plate from the asymptotic solution and the present solution 
with three boundary conditions along the two opposite edges

The energy flow analysis presented in this paper makes the conservative assumption that the joint is undamped. This requirement is not easily satisfied with ABAQUS software. Here, the complete finite element analysis of the energy response is first used to validate the accuracy of an alternative wave component solution [44] which can consider the damping effect of the joint. The damping loss factor for all structural components is chosen as 0.01 . Then the wave component solution is used to validate the presented energy flow analysis. The wave component solution of energy response is briefly given in Appendix $\mathrm{C}$, where the wave amplitudes needed in this solution are obtained by using the hybrid analytical wave and finite element formulation. Fig. 18 gives the energy response from the ABAQUS reference result and the wave component solution with only propagative wave components considered. The comparison shows good agreement and it can be concluded that the propagative wave components carry the total energy of the structure. 


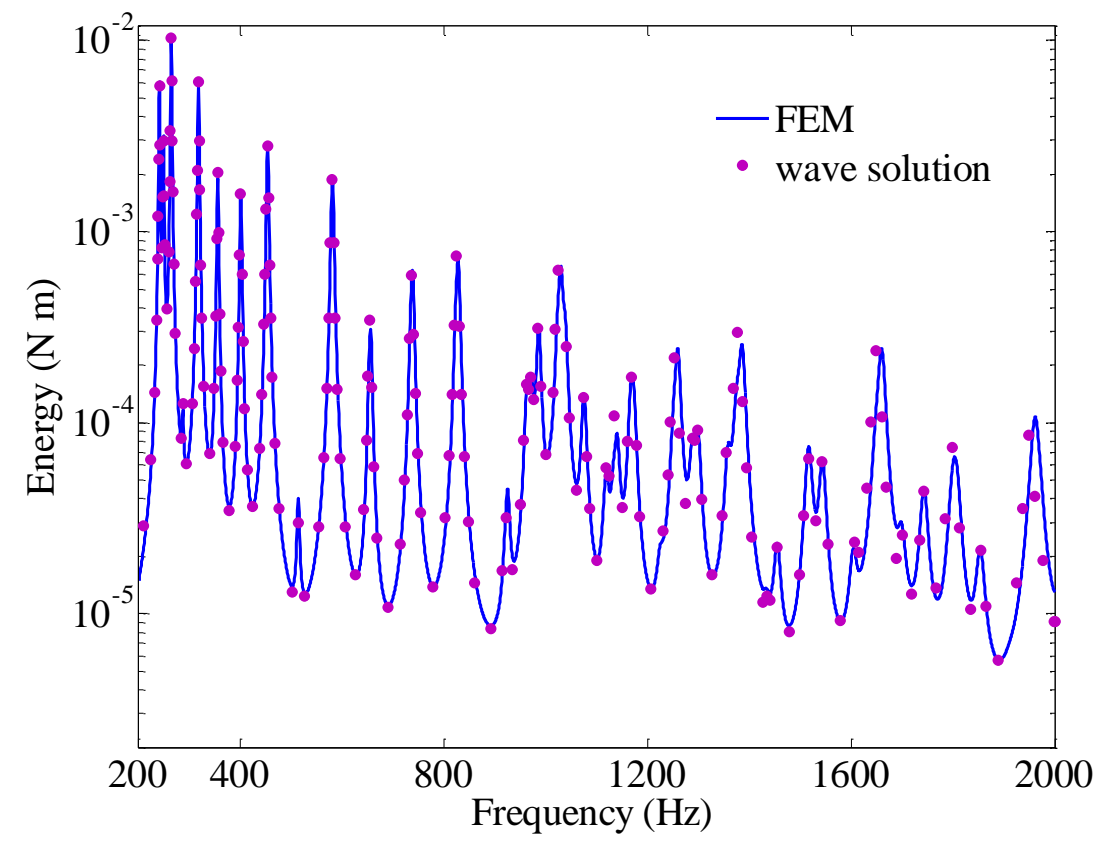

Fig. 18. Energy response from the ABAQUS reference result and the wave component solution of [44] with only propagative wave components considered.

The waves can spread energy and can be considered as subsystems only when they become propagative. Hence, analysis of the dispersion relation is necessary for the presented energy flow analysis. For the plate considered here, the authors have investigated the dispersion relation in [43], and found that the first and the second flexural waves become propagative at $241 \mathrm{~Hz}$ and $963 \mathrm{~Hz}$, respectively. Figs. 19 and 20 present the energy response of each plate from the presented method and the wave component solution. For plate 1, which is excited directly by the external force, good agreement is observed between the results of the two methods. For plate 2, which has no direct excitation from the external force, good agreement can be observed at all the peak values, but at the non-peak locations the present results are significantly larger than those from the wave component solution. 


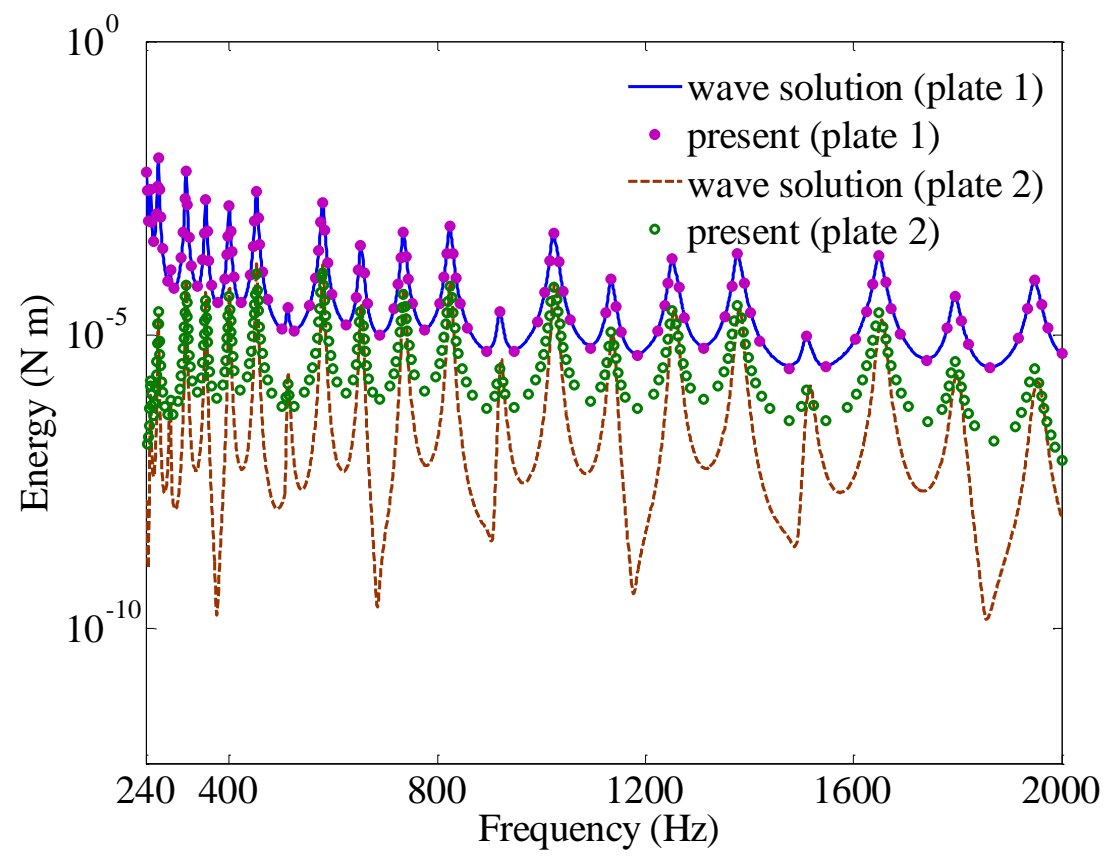

Fig. 19. Energy response of the first flexural wave from the presented method and the wave component solution.

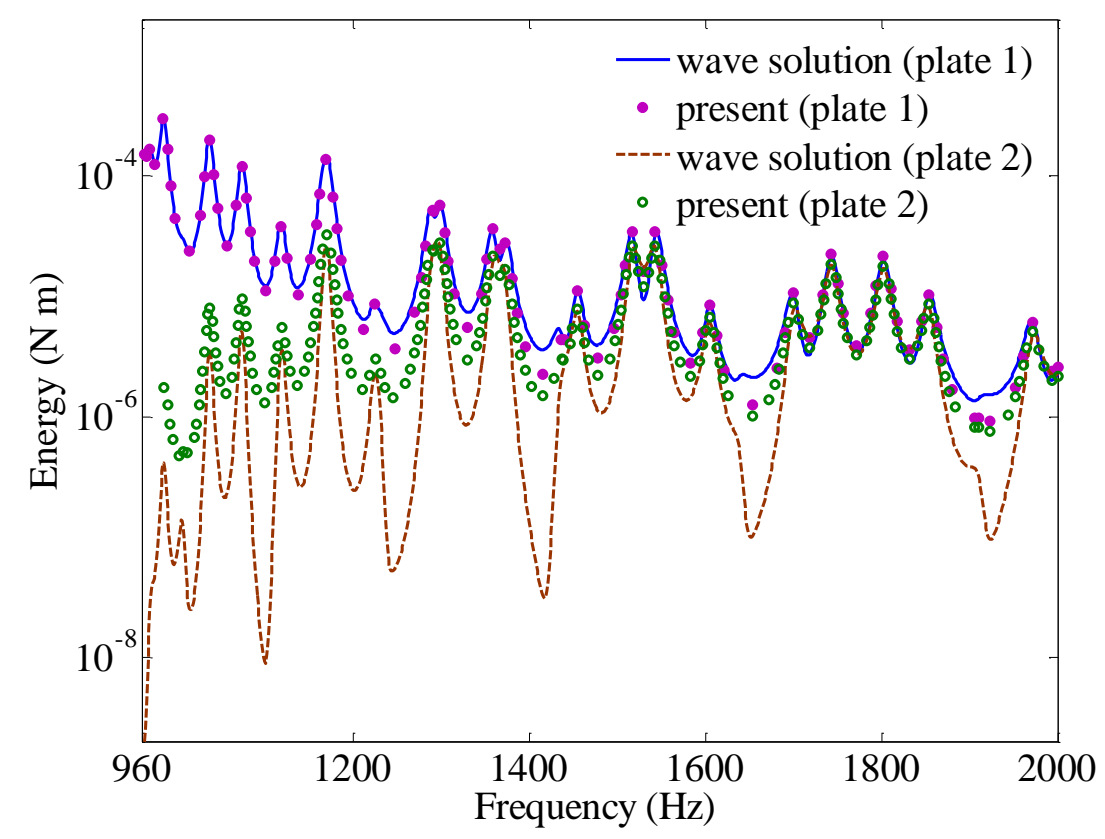

Fig. 20. Energy response of the second flexural wave from the presented method and the wave component solution. 
The effectiveness of Eq. (9) for evaluation of the coupling factor is validated next. Based on the concept of the power injection method (PIM) [33], an external force is applied on the two plates in turn, and the energy balance equations are expressed as

$$
\mathbf{C E}_{i}=\frac{1}{\omega} \Pi_{i}
$$

where $\mathbf{E}_{i}$ and $\boldsymbol{\Pi}_{i}(i=1,2)$ are the energy and input power of each wave subsystem calculated using the wave component solution. Thus

$$
\mathbf{C}\left[\begin{array}{ll}
\mathbf{E}_{1} & \mathbf{E}_{2}
\end{array}\right]=\frac{1}{\omega}\left[\begin{array}{ll}
\boldsymbol{\Pi}_{1} & \boldsymbol{\Pi}_{2}
\end{array}\right]
$$

from which $\mathbf{C}$ and subsequently the coupling factor can be obtained.

Fig. 21 gives the coupling factors associated with the first flexural wave subsystem from Eqs. (9) and (52). The blue heavy solid line is the result from Eq. (9), the gray thin solid lines are results from Eq. (52) considering various locations of the external force, and the heavy dashed line and the heavy dotted line are their averages, respectively. Good agreement can be observed for all the results at the peak values. But at non-peak locations, the results from Eq. (9) are significantly larger than the results from Eq. (52). This is because the form of Eq. (9) used in this paper is based on the resonant modes description used in classical SEA. Fig. 21 also matches the comparison in Fig. 19 that the two results agree well at peak values and clearly disagree at non-resonant locations. As can be seen from Fig. 21, the coupling factor associated with the first wave subsystem is much smaller than the damping loss factor (which has the value 0.01 ), indicating that the two plates are weakly coupled. Therefore, the significant disagreement of the coupling factor at the non-peak frequencies has little influence 
on the energy of the plate excited directly by the external force, as confirmed by Fig. 19 where its energy responses calculated by the two methods agree very well. It is also seen from Fig. 19 that the energy level at the peak values is significantly larger than that ather frequencies, and hence the disagreement of the energy levels calculated by the two methods at non-resonant locations will not reduce the practicability of the presented method.

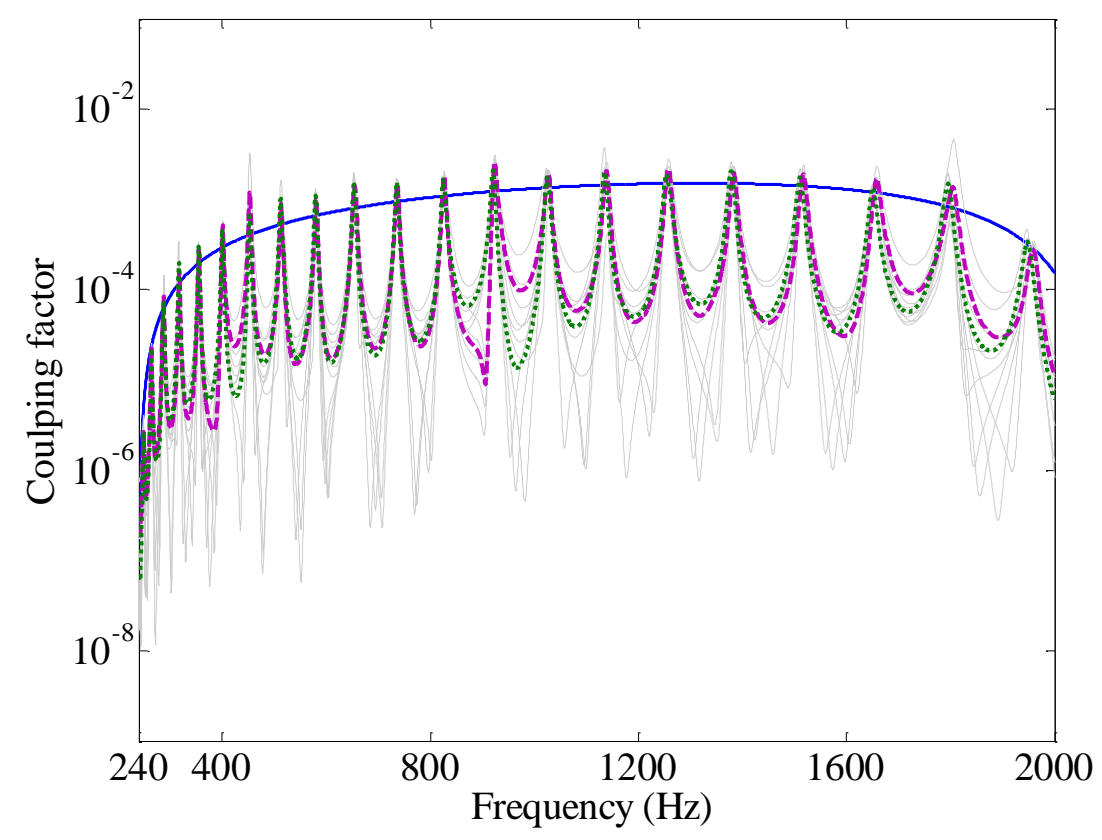

Fig. 21. Coupling factors associated with the first flexural wave subsystem from Eqs. (9) and (52); the blue heavy solid line is the result from Eq. (9), the gray thin solid lines are results from Eq. (52) considering various locations of the external force, and the heavy dashed line and the heavy dotted line are their averages respectively. 


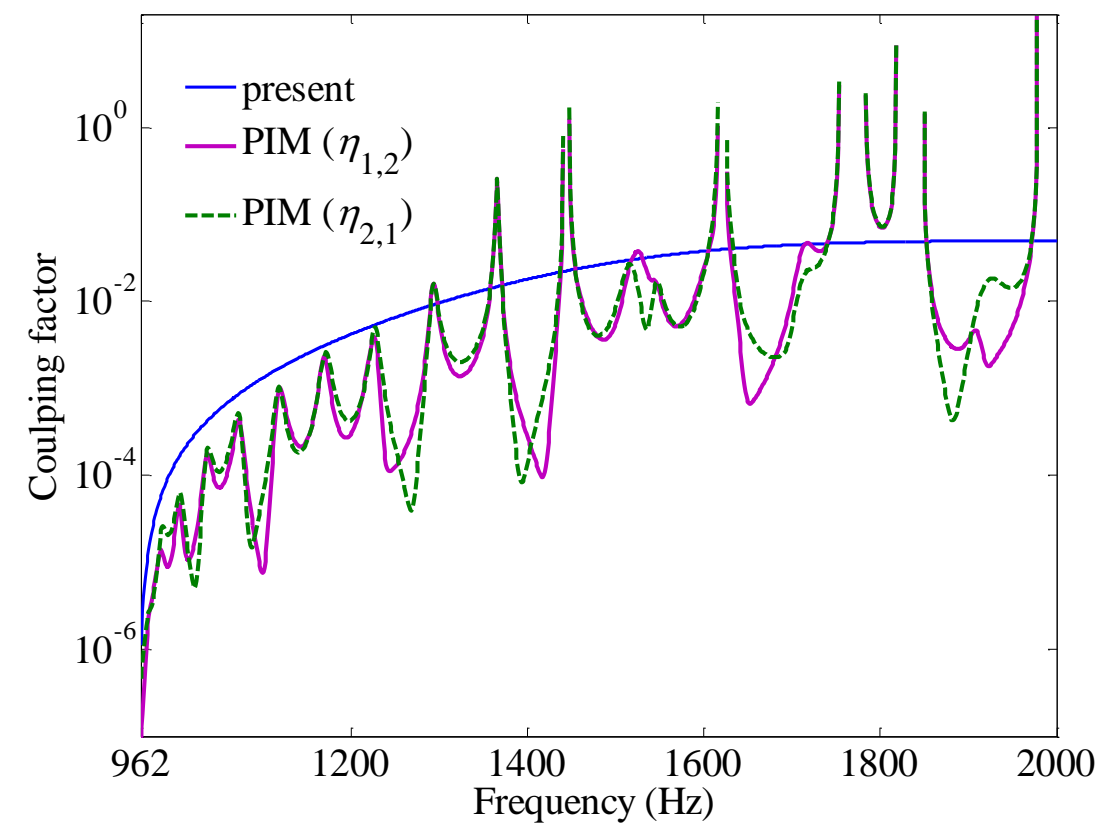

Fig. 22. Coupling factors associated with the second wave subsystem from Eqs. (9) and (52).

Fig. 22 shows the coupling factors associated with the second wave subsystem from Eqs. (9) and (52), with the latter based on one group of excitation locations. As can be seen, the results from the two methods disagree with each other above $1300 \mathrm{~Hz}$. Also, at some frequencies the results from Eq. (52) are negative which is incorrect. Comparing with Fig. 20, it can be concluded that the reason for this is that the energies of the second wave subsystems of the two plates are comparable at these frequencies. Hence when using Eq. (52) to evaluate the coupling factor, the inverse operation frequently suffers numerical ill-conditioning. As can be seen from Fig. 20, the second wave subsystems are strongly coupled above $1300 \mathrm{~Hz}$, which makes Eq. (52) no longer appropriate for evaluating the coupling factor. However, the energy of the second wave subsystem can still be evaluated accurately from Eq. (13), which means that the energy flow analysis presented in this paper is suitable not only for weakly coupled systems but also for strongly coupled systems. 
It is noticed that with increasing frequency the second wave subsystems of the two plates become strongly coupled while the first wave subsystem remains weakly coupled. This is attributed to the inherent characteristics of the example structure used here. The line at which the vertical deformation is prevented is placed exactly at the peak location of the first flexural wave shape, and this seriously hinders the energy transmission between the first wave subsystems of the two plates. Moreover, the energy transmission between the second wave subsystems of the two plates is not limited which makes them strongly coupled. Therefore, the fact that the energy of each wave subsystem can be directly obtained by the present analysis provides useful guidance for the control of structural vibration.

\subsubsection{Three angle coupled plates with a joint}

Based on the two plate structure in the previous example, a third plate is added along the line region with end points $(0 \mathrm{~m}, 0.015 \mathrm{~m})$ and $(0.1 \mathrm{~m}, 0.015 \mathrm{~m})$. The angle between the second and third plates is $45^{\circ}$. The geometry and material properties of the third plate are the same as those of the other two plates. Unlike the two plate structure, the contribution of the in-plane vibration of each plate must now be considered. The boundary conditions for flexural vibration are the same as those of the two plate structure. The in-plane boundary condition of each plate is SS1-SS2 [46]. The uncoupled end of each plate is free. The finite element model of the joint is the same as that used previously, except that the two ends are clamped.

Figs. 23 and 24 present the energy responses of the first and second wave subsystems of the three plates from the presented energy flow analysis and the wave component solution. For plate 1, which is directly excited by the external force, the results from the two methods 
agree with each other very well. But for plates 2 and 3, which have no direct excitation, only the results at peak values agree with each other and at the non-peak locations the results from the presented method are larger than those from the wave component solution. These conclusions are exactly the same as were observed for the two plate structure. It can also be found that, due to the addition of the third plate, unlike the strongly coupled relation in the two plate structure, the second wave subsystems of the first and second plates are always weakly coupled.

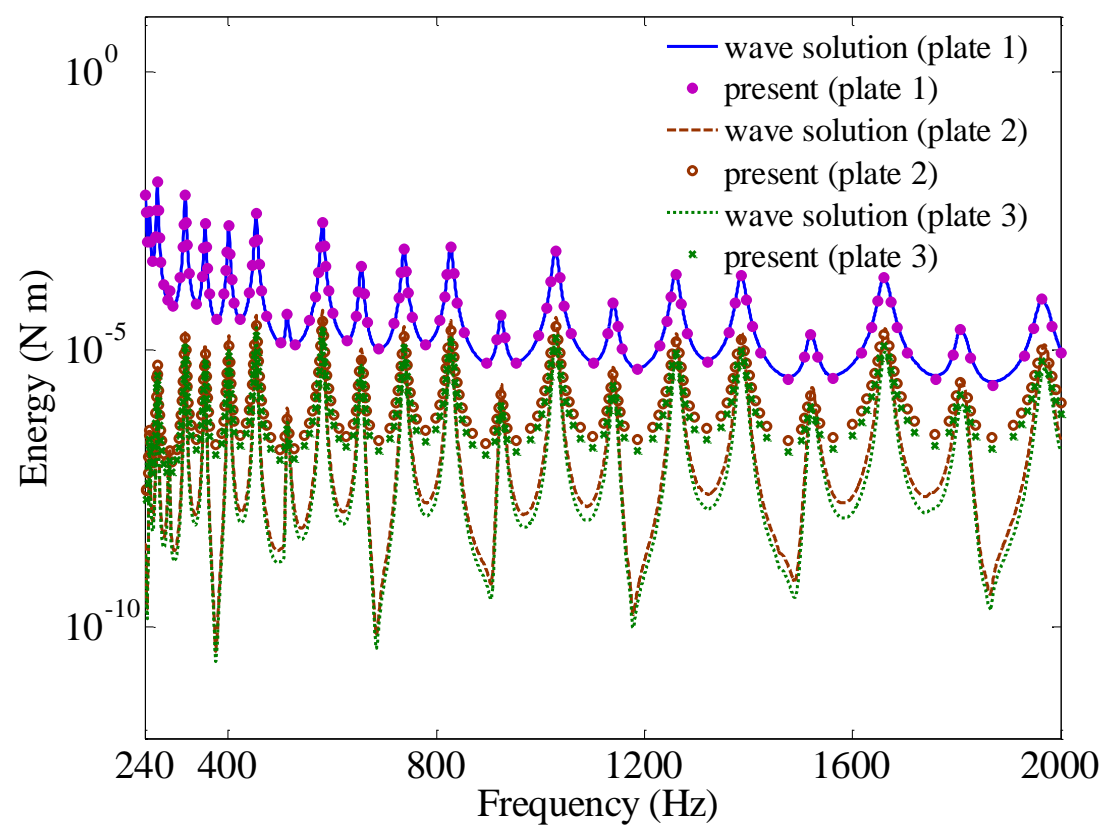

Fig. 23. Energy response of the first flexural wave from the presented method and the wave component solution. 


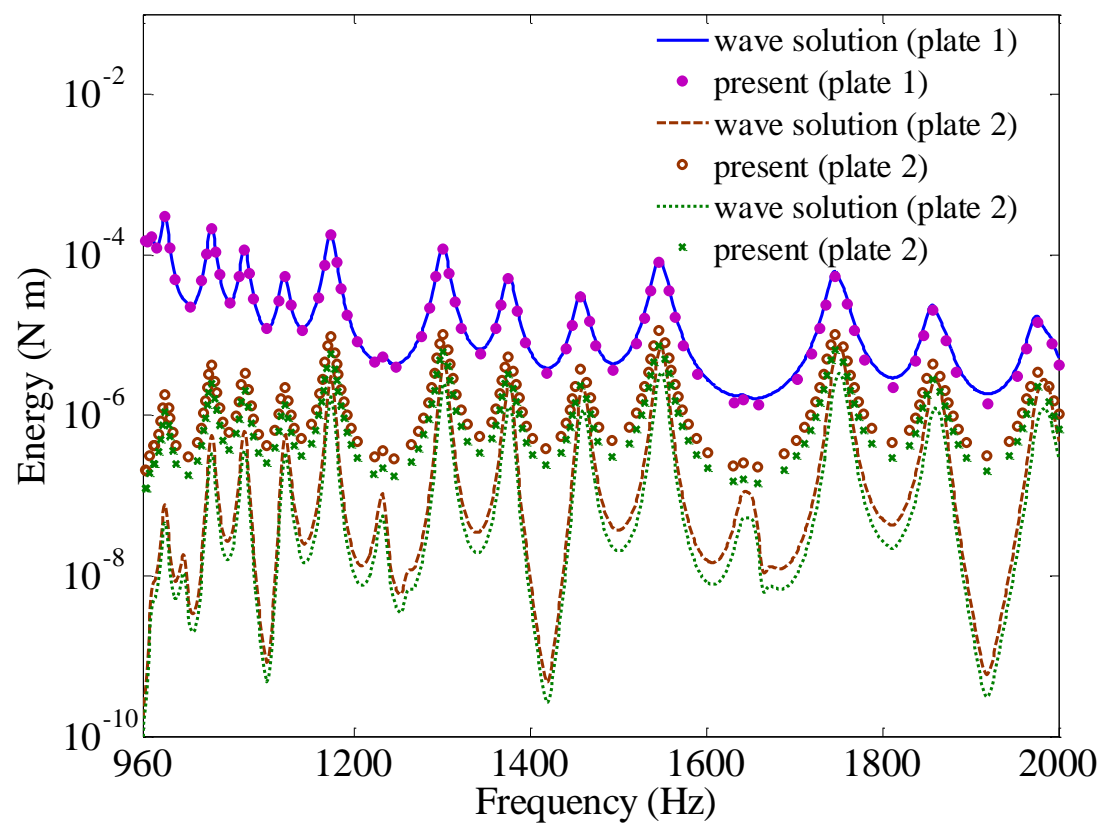

Fig. 24. Energy response of the second flexural wave from the presented method and the wave component solution.

\section{Conclusions}

Based on the balance relation between the input, transmission and dissipation of the energy of each subsystem, an energy flow analysis method with high accuracy and high efficiency is proposed in this paper for the mid-frequency vibration of built-up plate structures. The analysis parameters of mode count, modal density, damping and coupling loss factors and input mobility used in the classical SEA are well considered here, and the method presented can be regarded to some extent as an extension of the classical SEA into the mid-frequency region. Unlike the classical SEA, the propagative waves of each plate component are regarded as subsystems. Considering the power balance of each wave subsystem, the system matrix equation is established. The numerical examples show that the solution framework of energy flow balance of SEA can be successfully extended to the analysis of mid-frequency structural 
vibration. The highlights of the paper are summarized as follows.

1) The wave components are obtained by using the symplectic method, and hence arbitrary boundary conditions and therefore the mid-frequency characteristic of the plates can be exactly considered. The solutions for the mode count, modal density and group velocity of the plates can be transformed into solutions for these parameters of a one-dimensional wave subsystem, and symplectic analytical results can be obtained. The solution for the coupling factor is validated by comparison with results from the power injection method. Also, for strongly coupled systems where the power injection method fails to evaluate the coupling factor, however, the presented method can still provide coupling factors which reflect the mid-frequency characteristic of the structure.

2) Compared to FEM, the advantages of high accuracy and efficiency of the presented hybrid analytical wave and finite element formulation can be observed in evaluating the local response of the structure. The high efficiency of the hybrid formulation increases significantly with increasing plate length/width ratio. For analysis where the finite element model of the joint is relatively large, the modal expansion technique presented in this paper can significantly reduce the computational load of the inverse operation. In addition, the wave scattering property of the joint structure can be obtained from the hybrid formulation and can then replace the finite element model of the joint in the hybrid formulation when performing a repetition of the analysis. This practice will save much computational time, especially when investigating the dependency of the response on the form of the external force or the plate length.

3) In contrast with traditional displacement based methods for energy analysis, such as 
FEM and the wave component solution, the energy flow analysis presented in this paper uses the energy of each subsystem as degrees of freedom, and hence is of great computational efficiency. In contrast with SEA, since the energy of each wave subsystem is obtained directly, the vibrational transmission characteristics of the built-up structure can be understood more deeply to provide useful guidance for controlling the transmission of structural vibration.

\section{Acknowledgements}

The authors are grateful for support under grants from the National Science Foundation of China (11172056), the National Basic Research Program of China (2014CB046803), and the Cardiff University Advanced Chinese Engineering Centre.

\section{Appendix A. Derivations for the displacement-force relation at the plates' coupling interfaces}

For a plate whose left end is uncoupled, the boundary condition can be expressed as

$$
\mathbf{E}\left(\mathbf{A} \widehat{\mathbf{T}}(0)\left\{\begin{array}{l}
\mathbf{a}_{1}^{+} \\
\mathbf{a}_{\mathrm{r}}^{-}
\end{array}\right\}+\mathbf{A T}_{\mathbf{l}}(0) \mathbf{e}\right)=\mathbf{0}
$$

where $\mathbf{E}$ is the index matrix of the boundary condition. Without loss of generality, taking the free boundary condition as an example, $\mathbf{E}=\operatorname{diag}\{0,0,1,1\}$. From Eq. (A.1)

$$
\boldsymbol{\Theta} \widehat{\mathbf{T}}(0)\left\{\begin{array}{l}
\mathbf{a}_{1}^{+} \\
\mathbf{a}_{\mathrm{r}}^{-}
\end{array}\right\}+\boldsymbol{\Theta} \mathbf{T}_{1}(0) \mathbf{e}=\mathbf{0}
$$

where $\boldsymbol{\Theta}=\int_{0}^{a} \mathbf{A}_{\mathbf{x}}^{\mathrm{T}}(x) \mathbf{A}_{\mathbf{f}}(x) \mathrm{d} x$ and $a$ is the plate width. For in-plane vibration, $\mathbf{A}_{\mathbf{x}}=\left[\begin{array}{ll}\mathbf{A}_{u}^{+\mathrm{T}} & \mathbf{A}_{v}^{+\mathrm{T}}\end{array}\right]^{\mathrm{T}}, \quad \mathbf{A}_{\mathbf{f}}=\left[\begin{array}{ll}\mathbf{A}_{T}^{+\mathrm{T}} & \mathbf{A}_{N}^{+\mathrm{T}}\end{array}\right]^{\mathrm{T}} ;$ while for flexural vibration $\mathbf{A}_{\mathbf{x}}=\left[\begin{array}{ll}\mathbf{A}_{w}^{+\mathrm{T}} & \mathbf{A}_{\theta}^{+\mathrm{T}}\end{array}\right]^{\mathrm{T}}$, 
$\mathbf{A}_{\mathbf{f}}=\left[\begin{array}{ll}\mathbf{A}_{F}^{+\mathrm{T}} & \mathbf{A}_{M}^{+\mathrm{T}}\end{array}\right]^{\mathrm{T}}$. The subscripts $u, v, w, \theta, T, N, F$ and $M$ are used to indicate the displacement and force elements of the wave shape matrix.

Let $\mathbf{U}=\left[\begin{array}{ll}\mathbf{U}_{1} & \mathbf{U}_{2}\end{array}\right]=\boldsymbol{\Theta} \widehat{\mathbf{T}}(0), \quad \mathbf{V}=\boldsymbol{\Theta} \mathbf{T}_{1}(0) \mathbf{e}, \quad \mathbf{R}_{11}=-\left(\mathbf{U}_{1}\right)^{-1} \mathbf{U}_{2}, \quad \mathbf{R}_{12}=-\left(\mathbf{U}_{1}\right)^{-1} \mathbf{V}$. Then from Eq. (A.2)

$$
\mathbf{a}_{1}^{+}=\mathbf{R}_{11} \mathbf{a}_{\mathrm{r}}^{-}+\mathbf{R}_{12}
$$

The displacement and force at the right coupling end of the plate region can be expressed as

$$
\begin{gathered}
\overline{\mathbf{A}} \widehat{\mathbf{T}}(b)\left(\left\{\begin{array}{c}
\mathbf{R}_{\mathrm{l} 1} \\
\mathbf{I}
\end{array}\right\} \mathbf{a}_{\mathrm{r}}^{-}+\left\{\begin{array}{c}
\mathbf{R}_{12} \\
\mathbf{0}
\end{array}\right\}\right)+\overline{\mathbf{A}} \mathbf{T}_{\mathrm{r}}(b) \mathbf{e}=\overline{\mathbf{q}} \\
\boldsymbol{\Theta} \widehat{\mathbf{T}}(b)\left(\left\{\begin{array}{c}
\mathbf{R}_{\mathrm{l} 1} \\
\mathbf{I}
\end{array}\right\} \mathbf{a}_{\mathbf{r}}^{-}+\left\{\begin{array}{c}
\mathbf{R}_{12} \\
\mathbf{0}
\end{array}\right\}\right)+\boldsymbol{\Theta} \mathbf{T}_{\mathrm{r}}(b) \mathbf{e}=-\mathbf{F}
\end{gathered}
$$

where $\overline{\mathbf{A}}$ is the discrete displacement waveform matrix, $\mathbf{I}$ is a unit matrix, $\mathbf{F}=\int_{0}^{a} \mathbf{A}_{\mathbf{x}}(x) \mathbf{f}(x) \mathrm{d} x$ and $\mathbf{f}(x)$ is the force applied by the joint to the right coupling edge of the plate. From Eq. (A.5)

$$
\mathbf{a}_{\mathrm{r}}^{-}=\mathbf{P F}+\mathbf{P \Theta}\left(\widehat{\mathbf{T}}(b)\left\{\begin{array}{c}
\mathbf{R}_{12} \\
\mathbf{0}
\end{array}\right\}+\mathbf{T}_{\mathrm{r}}(b) \mathbf{e}\right)
$$

where $\mathbf{P}=-\left(\boldsymbol{\Theta} \widehat{\mathbf{T}}(b)\left\{\begin{array}{c}\mathbf{R}_{11} \\ \mathbf{I}\end{array}\right\}\right)^{-1}$. Substituting Eq. (A.6) into Eq. (A.4) gives

$$
\begin{aligned}
& \overline{\mathbf{q}}=\overline{\mathbf{A}} \widehat{\mathbf{T}}(b)\left\{\begin{array}{c}
\mathbf{R}_{\mathrm{l} 1} \\
\mathbf{I}
\end{array}\right\} \mathbf{P F} \\
& +\overline{\mathbf{A}} \widehat{\mathbf{T}}(b)\left\{\begin{array}{c}
\mathbf{R}_{\mathbf{l} 1} \\
\mathbf{I}
\end{array}\right\} \mathbf{P \Theta}\left(\widehat{\mathbf{T}}(b)\left\{\begin{array}{c}
\mathbf{R}_{\mathrm{l} 2} \\
\mathbf{0}
\end{array}\right\}+\mathbf{T}_{\mathrm{r}}(b) \mathbf{e}\right)+\overline{\mathbf{A}} \widehat{\mathbf{T}}(b)\left\{\begin{array}{c}
\mathbf{R}_{\mathrm{l} 2} \\
\mathbf{0}
\end{array}\right\}+\overline{\mathbf{A}} \mathbf{T}_{\mathrm{r}}(b) \mathbf{e}
\end{aligned}
$$

Eq. (A.7) is the displacement-force relation at the coupling edge of a plate whose left end is uncoupled.

A similar derivation can be applied to a plate whose right end is uncoupled. The wave reflection relation at the right end can be obtained as

$$
\mathbf{a}_{\mathrm{r}}^{-}=\mathbf{R}_{\mathrm{r} 1} \mathbf{a}_{1}^{+}+\mathbf{R}_{\mathrm{r} 2}
$$


and then the reflected waves and the displacement-force relation at the coupling edge are obtained as, respectively

$$
\begin{gathered}
\mathbf{a}_{1}^{+}=\mathbf{Q F}-\mathbf{Q \Theta}\left(\widehat{\mathbf{T}}(0)\left\{\begin{array}{c}
\mathbf{0} \\
\mathbf{R}_{\mathrm{r} 2}
\end{array}\right\}+\mathbf{T}_{1}(0) \mathbf{e}\right) \\
\overline{\mathbf{q}}=\overline{\mathbf{A}} \widehat{\mathbf{T}}(0)\left\{\begin{array}{c}
\mathbf{I} \\
\mathbf{R}_{\mathrm{r} 1}
\end{array}\right\} \mathbf{Q F} \\
-\overline{\mathbf{A}} \widehat{\mathbf{T}}(0)\left\{\begin{array}{c}
\mathbf{I} \\
\mathbf{R}_{\mathrm{r} 1}
\end{array}\right\} \mathbf{Q \Theta}\left(\widehat{\mathbf{T}}(0)\left\{\begin{array}{c}
\mathbf{0} \\
\mathbf{R}_{\mathrm{r} 2}
\end{array}\right\}+\mathbf{T}_{1}(0) \mathbf{e}\right)+\overline{\mathbf{A}} \widehat{\mathbf{T}}(0)\left\{\begin{array}{c}
\mathbf{0} \\
\mathbf{R}_{\mathrm{r} 2}
\end{array}\right\}+\overline{\mathbf{A}} \mathbf{T}_{1}(0) \mathbf{e}
\end{gathered}
$$

where $\mathbf{Q}=\left(\boldsymbol{\Theta} \widehat{\mathbf{T}}(0)\left\{\begin{array}{c}\mathbf{I} \\ \mathbf{R}_{\mathrm{r} 1}\end{array}\right\}\right)^{-1}$.

\section{Appendix B. The assembling of the displacement-force relation of each plate}

For convenience, the in-plane variables and flexural variables are put together, so that on the coupling interface of the $i$ th plate

$$
\overline{\mathbf{q}}_{i}=\left\{\begin{array}{c}
\overline{\mathbf{q}}_{i, \text { in }} \\
\overline{\mathbf{q}}_{i, \text { out }}
\end{array}\right\}, \quad \overline{\mathbf{f}}_{i}=\left\{\begin{array}{c}
\overline{\mathbf{f}}_{i, \text { in }} \\
\overline{\mathbf{f}}_{i, \text { out }}
\end{array}\right\}
$$

By combining Eqs. (24), (25) and (28), the displacement-force relation of each plate component can be expressed in global coordinates as

$$
\overline{\mathbf{q}}_{i, \mathrm{glob}}=\mathbf{h}_{i} \overline{\mathbf{f}}_{i, \mathrm{glob}}+\mathbf{g}_{i}
$$

where $\mathbf{h}_{i}=\boldsymbol{\Gamma}_{i}^{-1} \mathbf{R}\left[\begin{array}{cc}\tilde{\mathbf{h}}_{i, \text { in }} & \mathbf{0} \\ \mathbf{0} & \tilde{\mathbf{h}}_{i, \text { out }}\end{array}\right] \mathbf{R}^{-1} \boldsymbol{\Gamma}_{i}, \quad \mathbf{g}_{i}=\boldsymbol{\Gamma}_{i}^{-1} \mathbf{R}\left\{\begin{array}{c}\tilde{\mathbf{g}}_{i, \text { in }} \\ \tilde{\mathbf{g}}_{i, \text { out }}\end{array}\right\}, \quad \mathbf{R}$ is a position transform matrix of the degrees of freedom, $\boldsymbol{\Gamma}_{i}=\operatorname{diag}\left\{\tilde{\boldsymbol{\Gamma}}_{i, n}\right\}$ is the matrix of coordinate transformation, and 


$$
\tilde{\boldsymbol{\Gamma}}_{i, n}=\left[\begin{array}{cccc}
1 & 0 & 0 & 0 \\
0 & \cos \left(\varphi_{i}\right) & \sin \left(\varphi_{i}\right) & 0 \\
0 & -\sin \left(\varphi_{i}\right) & \cos \left(\varphi_{i}\right) & 0 \\
0 & 0 & 0 & 1
\end{array}\right]
$$

where $\varphi_{i}$ is the counterclockwise rotation angle of the local coordinate of each plate relative to the global coordinate. For plates whose left end is uncoupled,

$$
\begin{gathered}
\tilde{\mathbf{h}}=\overline{\mathbf{A}} \widehat{\mathbf{T}}(b)\left\{\begin{array}{c}
\mathbf{R}_{\mathrm{l} 1} \\
\mathbf{I}
\end{array}\right\} \mathbf{P} \overline{\mathbf{A}}_{\mathbf{x}} \\
\tilde{\mathbf{g}}=\overline{\mathbf{A}} \widehat{\mathbf{T}}(b)\left\{\begin{array}{c}
\mathbf{R}_{\mathbf{l} 1} \\
\mathbf{I}
\end{array}\right\} \mathbf{P \Theta}\left(\widehat{\mathbf{T}}(b)\left\{\begin{array}{c}
\mathbf{R}_{12} \\
\mathbf{0}
\end{array}\right\}+\mathbf{T}_{\mathrm{r}}(b) \mathbf{e}\right)+\overline{\mathbf{A}} \widehat{\mathbf{T}}(b)\left\{\begin{array}{c}
\mathbf{R}_{\mathrm{l} 2} \\
\mathbf{0}
\end{array}\right\}+\overline{\mathbf{A}} \mathbf{T}_{\mathrm{r}}(b) \mathbf{e}
\end{gathered}
$$

For plates whose right end is uncoupled

$$
\begin{gathered}
\tilde{\mathbf{h}}=\overline{\mathbf{A}} \widehat{\mathbf{T}}(0)\left\{\begin{array}{c}
\mathbf{I} \\
\mathbf{R}_{\mathrm{r} 1}
\end{array}\right\} \mathbf{Q} \overline{\mathbf{A}}_{\mathbf{x}} \\
\tilde{\mathbf{g}}=-\overline{\mathbf{A}} \widehat{\mathbf{T}}(0)\left\{\begin{array}{c}
\mathbf{I} \\
\mathbf{R}_{\mathrm{r} 1}
\end{array}\right\} \mathbf{Q \Theta}\left(\widehat{\mathbf{T}}(0)\left\{\begin{array}{c}
\mathbf{0} \\
\mathbf{R}_{\mathrm{r} 2}
\end{array}\right\}+\mathbf{T}_{\mathrm{l}}(0) \mathbf{e}\right)+\overline{\mathbf{A}} \widehat{\mathbf{T}}(0)\left\{\begin{array}{c}
\mathbf{0} \\
\mathbf{R}_{\mathrm{r} 2}
\end{array}\right\}+\overline{\mathbf{A}} \mathbf{T}_{1}(0) \mathbf{e}
\end{gathered}
$$

Assembling the equations of each plate according to Eq. (B.2) gives

$$
\overline{\mathbf{q}}_{\mathrm{c}}=\mathbf{H} \overline{\mathbf{f}}_{\mathrm{c}}+\mathbf{G}
$$

where $\mathbf{H}=\operatorname{diag}\left\{\mathbf{h}_{1}, \mathbf{h}_{2}, \ldots, \mathbf{h}_{N}\right\}, \mathbf{G}=\left[\mathbf{g}_{1}^{\mathrm{T}}, \mathbf{g}_{2}^{\mathrm{T}}, \ldots, \mathbf{g}_{N}^{\mathrm{T}}\right]^{\mathrm{T}}$

\section{Appendix C. Wave component solution for the energy and power flow}

The wave component solution for the amplitudes of energy and the power can be obtained using the hybrid formulation presented in this paper, together with results from [44].

The time average kinetic and strain energy stored in the plates are

$$
\left\langle E_{\mathrm{k}}\right\rangle=\frac{\rho h \omega^{2}}{4} \operatorname{Re}\left(\mathbf{a}^{\mathrm{H}} \int_{0}^{b} \widehat{\mathbf{T}}^{\mathrm{H}} \boldsymbol{\Omega}_{\mathrm{k}} \widehat{\mathbf{T}} \mathrm{d} y \mathbf{a}\right)+\frac{\rho h \omega^{2} \xi^{\mathrm{k}}}{4}
$$




$$
\left\langle E_{\mathrm{s}}\right\rangle=\frac{h}{4} \operatorname{Re}\left(\mathbf{a}^{\mathrm{H}} \int_{0}^{b} \widehat{\mathbf{T}}^{\mathrm{H}} \boldsymbol{\Omega}_{\mathrm{s}} \widehat{\mathbf{T}} \mathrm{d} y \mathbf{a}\right)+\frac{h \xi^{\mathrm{s}}}{4}
$$

respectively, where ()$^{\mathrm{H}}$ denotes the Hermitian transpose of a matrix; $\mathbf{\Omega}_{\mathrm{k}}=\int_{0}^{a} \mathbf{A}_{w}^{\mathrm{H}} \mathbf{A}_{w} \mathrm{~d} x$; $\boldsymbol{\Omega}_{\mathrm{s}}=\int_{0}^{a} \widetilde{\mathbf{A}}^{\mathrm{H}} \Xi \widetilde{\mathbf{A}} \mathrm{d} x ; \xi^{\mathrm{k}}$ and $\xi^{\mathrm{s}}$ are force relevant terms expressed as

$$
\begin{aligned}
& \xi^{\mathrm{i}}=\operatorname{Re}\left(\left[\begin{array}{ll}
\mathbf{a}_{1}^{+\mathrm{H}} & \mathbf{a}_{\mathrm{r}}^{-\mathrm{H}}
\end{array}\right]\left(\int_{0}^{y_{\mathrm{e}}} \widehat{\mathbf{T}}^{\mathrm{H}} \boldsymbol{\Omega}_{\mathrm{i}} \mathbf{T}_{\mathrm{l}} \mathrm{d} y+\int_{y_{\mathrm{e}}}^{b} \widehat{\mathbf{T}}^{\mathrm{H}} \boldsymbol{\Omega}_{\mathrm{i}} \mathbf{T}_{\mathrm{r}} \mathrm{d} y\right)\left[\begin{array}{ll}
\mathbf{e}^{+\mathrm{T}} & \mathbf{e}^{-\mathrm{T}}
\end{array}\right]^{\mathrm{T}}\right. \\
& +\left[\begin{array}{ll}
\mathbf{e}^{+\mathrm{H}} & \mathbf{e}^{-\mathrm{H}}
\end{array}\right]\left(\int_{0}^{y_{\mathrm{e}}} \mathbf{T}_{1}^{\mathrm{H}} \boldsymbol{\Omega}_{\mathrm{i}} \widehat{\mathbf{T}} \mathrm{d} y+\int_{y_{\mathrm{e}}}^{b} \mathbf{T}_{\mathrm{r}}^{\mathrm{H}} \boldsymbol{\Omega}_{\mathrm{i}} \widehat{\mathbf{T}} \mathrm{d} y\right)\left[\begin{array}{ll}
\mathbf{a}_{\mathrm{l}}^{+\mathrm{T}} & \mathbf{a}_{\mathrm{r}}^{-\mathrm{T}}
\end{array}\right]^{\mathrm{T}}
\end{aligned}
$$

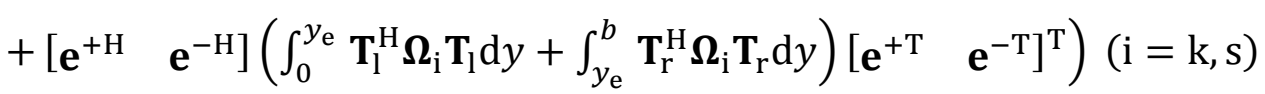

Details of $\widetilde{\mathbf{A}}$ and $\boldsymbol{\Xi}$ are given in [44].

The input power is expressed as

$$
\langle\Pi\rangle=-\frac{\omega}{2} \operatorname{Im}\left(\mathbf{a}^{\mathrm{H}} \boldsymbol{\Omega}_{\Pi} \mathbf{a}\right)
$$

where $\boldsymbol{\Omega}_{\Pi}=\int_{0}^{a}\left\{\mathbf{A}_{F}^{\mathrm{H}}(x) \mathbf{A}_{w}(x)+\mathbf{A}_{M}^{\mathrm{H}}(x) \mathbf{A}_{\theta}(x)\right\} \mathrm{d} x$.

\section{References}

[1] M. Petyt, Introduction to Finite Element Vibration Analysis, Cambridge University Press, Cambridge, 2010.

[2] C.A. Brebbia, J. Dominguez, Boundary Elements: An Introductory Course, WIT Press, Southampton, 1992.

[3] R.H. Lyon, R.G. DeJong, Theory and Application of Statistical Energy Analysis, Butterworth-Heinemann, Boston, 1995.

[4] C. Vanmaele, D. Vandepitte, W. Desmet, An efficient wave based prediction technique for plate bending vibrations. Computer Methods in Applied Mechanics and Engineering 196 (2007) 3178-3189. 
[5] L.F. Greengard, V. Rokhlin, A fast algorithm for particle simulations. Journal of Computational Physics 73 (1987) 325-348.

[6] S. Dey, J.J. Shirron, L.S. Couchman, Mid-frequency structural acoustic and vibration analysis in arbitrary, curved three-dimensional domains. Computers \& Structures 79 (2001) 617-629.

[7] T. Strouboulis, K. Copps, I. Babuška, The generalized finite element method. Computer Methods in Applied Mechanics and Engineering 190 (2001) 4081-4193.

[8] I. Harari, F. Magoules, Numerical investigations of stabilized finite element computations for acoustics. Wave Motion 39 (2004) 339-349.

[9] M. Bonnet, S. Chaillat, J.-F. Semblat, A multi-level fast multipole BEM for 3-D elastodynamics in the frequency domain. Computer Methods in Applied Mechanics and Engineering 197 (2008) 4233-4249.

[10]J.H. Ko, D. Byun, Comparison on numerical solutions for mid-frequency response analysis of finite element linear systems. Computers \& Structures 88 (2010) 18-24.

[11]A.N. Bercin, R.S. Langley, Application of the dynamic stiffness technique to the in-plane vibrations of plate structures. Computers \& Structures 59 (1996) 869-875.

[12]U. Orrenius, S. Finnveden, Calculation of wave propagation in rib-stiffened plate structures. Journal of Sound and Vibration 198 (1996) 203-224.

[13]B.R. Mace, D. Duhamel, M.J. Brennan, L. Hinke, Finite element prediction of wave motion in structural waveguides. Journal of the Acoustical Society of America 117 (2005) $2835-2843$.

[14]J.M. Mencik, M.N. Ichchou, Multi-mode propagation and diffusion in structures through 
finite elements. European Journal of Mechanics-A/Solids 24 (2005) 877-898.

[15] Y. Chen, G. Jin, M. Zhu, Z. Liu, J. Du, W. Li, Vibration behaviors of a box-type structure built up by plates and energy transmission through the structure. Journal of Sound and Vibration 331 (2012) 849-867.

[16]K. Vergote, C. Vanmaele, D. Vandepitte, W. Desmet, An efficient wave based approach for the time-harmonic vibration analysis of 3D plate assemblies. Journal of Sound and Vibration 332 (2013) 1930-1946.

[17]H. Riou, P. Ladevèze, L. Kovalevsky, The variational theory of complex rays: an answer to the resolution of mid-frequency 3D engineering problems. Journal of Sound and Vibration 332 (2013) 1947-1960.

[18]R.S. Langley, A wave intensity technique for the analysis of high frequency vibrations. Journal of Sound and Vibration 159 (1992) 483-502.

[19]D.J. Nefske, S.H. Sung, Power flow finite element analysis of dynamic systems: basic theory and application to beams. Journal of Vibration Acoustics Stress and Reliability in Design 111 (1989) 94-100.

[20]N. Vlahopoulos, X. Zhao, T. Allen, An approach for evaluating power transfer coefficients for spot-welded joints in an energy finite element formulation. Journal of Sound and Vibration 220 (1999) 135-154.

[21]L. Maxit, J.L. Guyader, Extension of SEA model to subsystems with non-uniform modal energy distribution. Journal of Sound and Vibration 265 (2003) 337-358.

[22]X. Zhao, N. Vlahopoulos, A hybrid finite element formulation for mid-frequency analysis of systems with excitation applied on short members. Journal of Sound and Vibration 237 
(2000) 181-202.

[23]P.J. Shorter, R.S. Langley, Vibro-acoustic analysis of complex systems. Journal of Sound and Vibration 288 (2005) 669-699.

[24]L. Ji, B.R. Mace, R.J. Pinnington, A mode-based approach for the mid-frequency vibration analysis of coupled long-and short-wavelength structures. Journal of Sound and Vibration 289 (2006) 148-170.

[25]R.S. Langley, J.A. Cordioli, Hybrid deterministic-statistical analysis of vibro-acoustic systems with domain couplings on statistical components. Journal of Sound and Vibration 321 (2009) 893-912.

[26]K. Vergote, B. Van Genechten, D. Vandepitte, W. Desmet, On the analysis of vibro-acoustic systems in the mid-frequency range using a hybrid deterministic-statistical approach. Computers \& Structures 89 (2011) 868-877.

[27]R.H. Lyon, G. Maidanik, Power flow between linearly coupled oscillators. Journal of the Acoustical Society of America 34 (1962) 623-639.

[28]T. Lafont, N. Totaro, A. Le Bot, Review of statistical energy analysis hypotheses in vibroacoustics. Proceedings of the Royal Society of London A: Mathematical, Physical and Engineering Sciences 470 (2014) 20130515

[29]G. Xie, D.J. Thompson, C.J.C. Jones, Mode count and modal density of structural systems: relationships with boundary conditions. Journal of Sound and Vibration 274 (2004) 621-651.

[30]A. Seçgin, Numerical determination of statistical energy analysis parameters of directly coupled composite plates using a modal-based approach. Journal of Sound and Vibration 
332 (2013) 361-377.

[31]S. Finnveden, Evaluation of modal density and group velocity by a finite element method. Journal of Sound and Vibration 273 (2004) 51-75.

[32]N. Totaro, C. Dodard, J.L. Guyader, SEA coupling loss factors of complex vibro-acoustic systems. Journal of Vibration and Acoustics 131 (2009) 041009.

[33]D.A. Bies, S. Hamid, In situ determination of loss and coupling loss factors by the power injection method. Journal of Sound and Vibration 70 (1980) 187-204.

[34]R.S. Langley, K.H. Heron, Elastic wave transmission through plate/beam junctions. Journal of Sound and Vibration 143 (1990) 241-253.

[35]C. Simmons, Structure-borne sound transmission through plate junctions and estimates of SEA coupling loss factors using the finite element method. Journal of Sound and Vibration 144 (1991) 215-227.

[36] J.A. Steel, R.J.M. Craik, Statistical energy analysis of structure-borne sound transmission by finite element methods. Journal of Sound and Vibration 178 (1994) 553-561.

[37]C. Hopkins, Statistical energy analysis of coupled plate systems with low modal density and low modal overlap. Journal of Sound and Vibration 251 (2002) 193-214.

[38]A.N. Thite, B.R. Mace, Robust estimation of coupling loss factors from finite element analysis. Journal of Sound and Vibration 303 (2007) 814-831.

[39]B.R. Mace, The statistical energy analysis of two continuous one-dimensional subsystems. Journal of Sound and Vibration 166 (1993) 429-461.

[40]E.C.N. Wester, B.R. Mace, Statistical energy analysis of two edge-coupled rectangular plates: ensemble averages. Journal of Sound and Vibration 193 (1996) 793-822. 
[41]W.A. Yao, W.X. Zhong, C.W. Lim, Symplectic Elasticity, World Scientific, Singapore, 2009.

[42]W.X. Zhong, F.W. Williams, Wave problems for repetitive structures and symplectic mathematics. Proceedings of the Institution of Mechanical Engineers, Part C: Journal of Mechanical Engineering Science 206 (1992) 371-379.

[43] Y.B. Ma, Y.H. Zhang, D. Kennedy, A symplectic analytical wave based method for the wave propagation and steady state forced vibration of rectangular thin plates. Journal of Sound and Vibration 339 (2015) 196-214.

[44] Y.B. Ma, Y.H. Zhang, Forced vibration and energy flow analysis of plate assemblies in symplectic space, Vulnerability, Uncertainty, and Risk: Quantification, Mitigation, and Management, ASCE (2014) 485-495.

[45]C.W. Lim, X.S. Xu, Symplectic elasticity: theory and applications, Applied Mechanics Reviews 63 (2010) 050802.

[46]D.J. Gorman, Exact solutions for the free in-plane vibration of rectangular plates with two opposite edges simply supported. Journal of Sound and Vibration 294 (2006) 131-161.

[47]DS Simulia Inc (2010), ABAQUS standard manual (version 6.10). 